\title{
BLOW-UP PHENOMENA FOR LINEARLY PERTURBED YAMABE PROBLEM ON MANIFOLDS WITH UMBILIC BOUNDARY
}

\author{
MARCO GHIMENTI, ANNA MARIA MICHELETTI, AND ANGELA PISTOIA
}

\begin{abstract}
We build blowing-up solutions for linear perturbation of the Yamabe problem on manifolds with umbilic boundary, provided the Weyl tensor is nonzero everywhere on the boundary and the dimension of the manifold is $n \geq 11$.
\end{abstract}

\section{INTRODUCTION}

The well known Yamabe problem consists of finding a constant scalar curvature metric which is pointwise conformal to a given metric $g$ on an $n$-dimensional $(n \geq 3)$ compact Riemannian manifold $M$ without boundary. From a PDE's point of view, this is equivalent to finding a positive solution to the semilinear elliptic equation

$$
L_{g} u=\kappa u^{\frac{n+2}{n-2}} \text { in } M
$$

where $\kappa$ is a constant, $L_{g} u=-\Delta_{g} u+c(n) R_{g} u$ is the conformal Laplacian for $g$ with scalar curvature $R_{g}$ and $c(n):=\frac{n-2}{4(n-1)}$. Indeed, if $u$ is a positive solution of (11), then the new metric $\tilde{g}=u^{\frac{4}{n-2}} g$ has scalar curvature $c(n) \kappa$.

This problem has been complete solved through the combined works of Yamabe [28], Trudinger [27, Aubin [4] and Schoen [26]. The structure of the full set of solutions of (11) has also been completely understood. We quote the survey of Brendle and Marques [6] for a complete overview on the compactness and noncompactness results. A related issue is the compactness of linear or non-linear perturbations of problem (1) which has been largely studied in the last few years with contributions by several authors (see [8, 9, 12, 13, 22, 23, 24, 25]).

An obvious extension of such problems is to consider manifolds with boundary. The Yamabe problem on manifolds with boundary was initially investigated by Escobar [10, 11]. In this case one would like to find a metric $g$ on an $n$-dimensional $(n \geq 3)$ compact Riemannian manifold $M$ with boundary $\partial M$ which has not only constant scalar curvature but constant mean curvature as well. This problem is equivalent to showing the existence of a positive solution to the boundary value problem

$$
\left\{\begin{array}{cc}
L_{g} u=\kappa u^{\frac{n+2}{n-2}} & \text { in } M \\
\partial_{\nu_{g}} u+\frac{n-2}{2} h_{g} u=\mathfrak{c} u^{\frac{n}{n-2}} & \text { on } \partial M
\end{array}\right.
$$

where $\nu_{g}$ is the unit outer normal and $h_{g}$ is the mean curvature. If such a solution exists, then the metric $\tilde{g}=u^{\frac{4}{n-2}} g$ has scalar curvature $c(n) \kappa$ and the boundary has mean curvature $\mathfrak{c}$. Problem (2) has been solved starting from Escobar in [10, 11] with contributions from several authors when either $\kappa \neq 0$ and $\mathfrak{c}=0$ or $\kappa=0$

1991 Mathematics Subject Classification. 35J60, 53C21.

Key words and phrases. Yamabe problem, manifold with umbilic boundary, linear perturbation, bubbling phenomena, Weyl tensor.

The first author is partially supported by P.R.A., University of Pisa. 
and $\mathfrak{c} \neq 0$ (see the recent paper by Disconzi and Khuri [7] for an exhaustive list of references)

In this paper we will focus on the zero scalar curvature case, i.e. $\kappa=0$, so problem (2) reduces to finding a positive solution to the boundary value problem

$$
\left\{\begin{array}{cc}
L_{g} u=0 & \text { in } M \\
\partial_{\nu} u+\frac{n-2}{2} h_{g} u=\mathfrak{c} u^{\frac{n}{n-2}} & \text { on } \partial M .
\end{array}\right.
$$

Solutions to (3) are critical points of the functional

$$
Q(u):=\frac{\int_{M}\left(|\nabla u|^{2}+\frac{n-2}{4(n-1)} R_{g} u^{2}\right) d v_{g}+\int_{\partial M} \frac{n-2}{2} h_{g} u^{2} d \sigma_{g}}{\left(\int_{\partial M}|u|^{\frac{2(n-1)}{n-2}} d \sigma\right)^{\frac{n-2}{n-1}}}, u \in H
$$

where $d v_{g}$ and $d \sigma_{g}$ denote the volume forms on $M$ and $\partial M$, respectively, and the space

$$
H:=\left\{u \in H_{g}^{1}(M): u \neq 0 \text { on } \partial M\right\} .
$$

Escobar in [10] introduced the Sobolev quotient

$$
Q(M, \partial M):=\inf _{H} Q(u),
$$

which is conformally invariant and always satisfies

$$
Q(M, \partial M) \leq Q\left(\mathbb{B}^{n}, \partial \mathbb{B}^{n}\right),
$$

where $\mathbb{B}^{n}$ is the unit ball in $\mathbb{R}^{n}$ endowed with the euclidean metric $\mathfrak{g}_{0}$. Following Aubin's approach (see [4]), Escobar proved that if $Q(M, \partial M)$ is finite and the strict inequality in (5) holds, i.e.

$$
Q(M, \partial M)<Q\left(\mathbb{B}^{n}, \partial \mathbb{B}^{n}\right),
$$

then the infimum (44) is achieved and a solution to problem (3) does exist. In the negative case, i.e. $Q(M, \partial M) \leq 0$, it is quite easy to prove that (6) holds. The positive case, i.e. $Q(M, \partial M)>0$, is the most difficult one and the proof of the validity of (6) required a lot of work. When $(M, g)$ is not conformally equivalent to $\left(\mathbb{B}^{n}, \mathfrak{g}_{0}\right)$, (6) has been proved by Escobar in [10, by Marques in [19, 20] and by Almaraz in 3 .

Once the existence of solutions of problem (3) is settled, a natural question concerns the structure of the full set of positive solutions of (3). If $Q(M, \partial M)<0$ the solution is unique and if $Q(M, \partial M)=0$ the solution is unique up to a constant factor. If $Q(M, \partial M)>0$ the situation turns out to be more delicate. Indeed, the round hemisphere provides the canonical example of non compactness, while compactness was proved by Felli and Ould-Ahmedou in [14] when $(M, g)$ is locally conformally flat and $\partial M$ is umbilic and by Almaraz in [1], when $n \geq 7$ and the tracefree second fundamental form of $\partial M$ is non zero everywhere. Up to our knowledge, the only non-compactness result is due to Almaraz in [2], where he constructs a sequence of blowing-up conformal metrics with zero scalar curvature and constant boundary mean curvature on a ball of dimension $n \geq 25$. It is unknown if the dimension 25 is sharp for the compactness, namely if $n \leq 24$ the problem (3) is compact or not.

The compactness issue is closely related to the existence of blowing-up solutions for small perturbations of problem (3). In particular, we consider the linear perturbation problem

$$
\left\{\begin{array}{cc}
-\Delta_{g} v+\frac{n-2}{4(n-1)} R_{g} v=0 & \text { in } M \\
\frac{\partial v}{\partial \nu}+\frac{n-2}{2} h_{g} v+\varepsilon \gamma v=(n-2) v^{\frac{n}{n-2}} & \text { on } \partial M
\end{array}\right.
$$


where $\varepsilon$ is a small positive parameter and $\gamma$ is a given smooth function, and we address the following question:

(Q) Does problem (7) have a family of solutions which blows up at one point of the manifold as $\varepsilon$ approaches zero?

A first positive answer was given by the authors in 16 when $n \geq 7$ and the boundary is not umbilic. In the present paper, we give a positive answer when the boundary is umbilic. Our main result reads as follows.

Theorem 1. Let $(M, g)$ be a smooth, $n$-dimensional Riemannian manifold of positive type with regular umbilic boundary $\partial M$. Suppose that $n \geq 11$ and that the Weyl tensor is not vanishing on $\partial M$. Let $\gamma: M \rightarrow \mathbb{R}$ a smooth function, $\gamma>0$ on $\partial M$. Then, for $\varepsilon>0$ small there exists a positive solution $v_{\varepsilon}$ of the problem (7) such that $v_{\varepsilon}$ blows up at a suitable point $q_{0} \in \partial M$ as $\varepsilon \rightarrow 0$.

Let us make some comments on our result.

(1) The proof relies on the classical finite dimensional Ljapunov-Schmidt procedure which has been successfully used in studying blowing-up phenomena in Yamabe type problems. However, here the umbilicity of the boundary forces us to deal with higher order terms in the expansion of the metric $g$, which makes the proof of the result technically harder than the one in [16].

(2) Our theorem does not provide the precise location of the blow-up point, because the explicit solution to linear problem (19) is necessary and this is far from being possible. Actually, it would be really interesting to detect the geometric function whose critical points generate the blowing-up solutions.

(3) We believe that the result holds true if $\gamma$ is positive somewhere (and not necessarily positive everywhere in $\partial M$ ) as suggested by Remark 11, where we exhibit a smooth function $\gamma$ which is not necessarily everywhere positive, for which problem (7) has a family of blowing-up solutions. Actually, we strongly believe that if $\gamma$ is negative everywhere there are no blowing-up solutions as $\varepsilon$ approaches 0 , i.e. the problem (7) is compact.

(4) Our ideas can be also applied to study the non-linear perturbation problem

$$
\left\{\begin{array}{cc}
-\Delta_{g} v+\frac{n-2}{4(n-1)} R_{g} v=0 & \text { in } M \\
\frac{\partial v}{\partial \nu}+\frac{n-2}{2} h_{g} v=(n-2) v^{\frac{n}{n-2}+\varepsilon} & \text { on } \partial M
\end{array}\right.
$$

In particular, we can extend the results of the authors in [15] to the geometric problem (8).

The proof of the result relies on a finite dimensional Ljapunov-Schmidt reduction, which is carried out as usual through different steps: first we find a good approximated solution (Section 2), next we reduce the problem to a finite dimensional one (Section 3), then we study the reduced problem (Section 4) and finally we complete the proof of Theorem 1 (Section 5).

\section{Preliminaries AND VARiational FRAMEWORK}

Notations. We collect here our main notations. We will use the indices $1 \leq$ $i, j, k, m, p, r, s \leq n-1$ and $1 \leq a, b, c, d \leq n$. We denote by $g$ the Riemannian metric, by $R_{a b c d}$ the full Riemannian curvature tensor, by $R_{a b}$ the Ricci tensor and by $R_{g}$ the scalar curvature of $(M, g)$; moreover the Weyl tensor of $(M, g)$ will be denoted by $W_{g}$.

Let $\left(h_{i j}\right)_{i j}(q)$ be the tensor of the second fundamental form in a point $q \in \partial M$. We recall that the boundary $\partial M$ is umbilic (i.e. composed only of umbilic points) when, for all $q \in \partial M, h_{i j}(q)=0$ for all $i \neq j$ and $h_{i i}(q)=h_{g}(q), h_{g}(q)$ being the mean curvature of $\partial M$ at the point $q$. 
The bar over an object (e.g. $\bar{W}_{g}$ ) will mean the restriction to this object to the metric of $\partial M$. By $-\Delta_{g}$ we denote the Laplace-Beltrami operator on $(M, g)$ and we will often use the common notation for conformal Laplacian $L_{g}=-\Delta_{g}+\frac{n-2}{4(n-1)} R_{g}$ and the conformal boundary operator $B_{g}=\frac{\partial}{\partial \nu}+\frac{n-2}{2} h_{g}$, where $\nu$ is the outward normal to $\partial M$. When we derive a tensor, e.g. $T_{i j}$, with respect to a coordinate $y_{l}$ we use the usual shortened notation $T_{i j, l}$ for $\frac{\partial}{\partial y_{l}} T_{i j}$.

Remark 2. Since $\partial M$ is umbilic for any $q \in \partial M$, there exists a metric $\tilde{g}_{q}=\tilde{g}$, conformal to $g, \tilde{g}_{q}=\Lambda_{q}^{\frac{4}{n-2}} g_{q}$ such that

$$
\begin{aligned}
\left|\operatorname{det} \tilde{g}_{q}(y)\right|=1+O\left(|y|^{n}\right) & \left|\tilde{h}_{i j}(y)\right|=o\left(\left|y^{3}\right|\right) \\
\tilde{g}^{i j}(y)= & \delta_{i j}+\frac{1}{3} \bar{R}_{i k j l} y_{k} y_{l}+R_{n i n j} y_{n}^{2} \\
+ & \frac{1}{6} \bar{R}_{i k j l, m} y_{k} y_{l} y_{m}+R_{n i n j, k} y_{n}^{2} y_{k}+\frac{1}{3} R_{n i n j, n} y_{n}^{3} \\
+ & \left(\frac{1}{20} \bar{R}_{i k j l, m p}+\frac{1}{15} \bar{R}_{i k s l} \bar{R}_{j m s p}\right) y_{k} y_{l} y_{m} y_{p} \\
+ & \left(\frac{1}{2} R_{n i n j, k l}+\frac{1}{3} \operatorname{Sym}_{i j}\left(\bar{R}_{i k s l} R_{n s n j}\right)\right) y_{n}^{2} y_{k} y_{l} \\
+ & \frac{1}{3} R_{n i n j, n k} y_{n}^{3} y_{k}+\frac{1}{12}\left(R_{n i n j, n n}+8 R_{n i n s} R_{n s n j}\right) y_{n}^{4}+O\left(|y|^{5}\right) \\
& \bar{R}_{\tilde{g}_{q}}(y)=O\left(|y|^{2}\right) \text { and } \partial_{i i}^{2} \bar{R}_{\tilde{g}_{q}}(q)=-\frac{1}{6}|\bar{W}(q)|^{2} \\
& \bar{R}_{k l}(q)=R_{n n}(q)=R_{n k}(q)=0
\end{aligned}
$$

uniformely with respect to $q \in M$ and $y \in T_{q}(M)$. Also,we have $\Lambda_{q}(q)=1$ and $\nabla \Lambda_{q}(q)=0$. This results are contained in [19, 17].

The conformal Laplacian and the conformal boundary operator transform under the change of metric $\tilde{g}_{q}=\Lambda_{q}^{\frac{4}{n-2}} g_{q}$ in the following way:

$$
\begin{aligned}
& L_{\tilde{g}_{q}} \varphi=\Lambda_{q}^{-\frac{n+2}{n-2}} L_{g}\left(\Lambda_{q} \varphi\right) \\
& B_{\tilde{g}_{q}} \varphi=\Lambda_{q}^{-\frac{n}{n-2}} B_{g}\left(\Lambda_{q} \varphi\right)
\end{aligned}
$$

by these transformations we can recast Problem (7) as follows: $v:=\Lambda_{q} u$ is a positive solution of (7), if and only if $u$ is a positive solution of

$$
\left\{\begin{array}{cc}
L_{\tilde{g}_{q}} u=0 & \text { in } M \\
B_{\tilde{g}_{q}} u+\varepsilon\left[\Lambda_{q}^{-\frac{2}{n-2}} \gamma\right] u=(n-2) u^{\frac{n}{n-2}} & \text { on } \partial M
\end{array}\right.
$$

From now on we set $\tilde{\gamma}=\Lambda_{q}^{-\frac{2}{n-2}} \gamma$.

We want to find a solution $u$ of problem (14) by a finite dimensional reduction: we will look for a solution of (14) of the form $u=W_{\delta, q}+\delta^{2} V_{\delta, q}+\Phi$ where $W_{\delta, q}$ and $V_{\delta, q}$ are functions depending only by $q \in \partial M$ and $\delta>0$ which will be defined in the following and $\Phi$ is a suitable remainder term. So we will find a solution of the original problem (7) of the type

$$
v=\Lambda_{q}\left[W_{\delta, q}+\delta^{2} V_{\delta, q}+\Phi\right] .
$$

In the following we simply use $\tilde{W}_{\delta, q}, \tilde{V}_{\delta, q}, \tilde{\Phi}$ respectively for $\Lambda_{q} W_{\delta, q}, \Lambda_{q} V_{\delta, q}, \Lambda_{q} \Phi$. 
If $Q(M, \partial M)>0$, we can endow $H_{g}^{1}(M)=H^{1}(M)$ with the following equivalent scalar product

$$
\langle\langle u, v\rangle\rangle_{g}=\int_{M}\left(\nabla_{g} u \nabla_{g} v+\frac{n-2}{4(n-1)} R_{g} u v\right) d \mu_{g}+\frac{n-2}{2} \int_{\partial M} h_{g} u v d \nu_{g}
$$

which leads to the norm $\|\cdot\|_{g}$ equivalent to the usual one. We remark also that $\Lambda_{q}$ is an isometry in the sense that, by (15), for $u, v \in H^{1}(M)$

$$
\left\langle\left\langle\Lambda_{q} u, \Lambda_{q} v\right\rangle\right\rangle_{g}=\langle\langle u, v\rangle\rangle_{\tilde{g}_{q}} \text { and, consequently, }\left\|\Lambda_{q} u\right\|_{g}=\|u\|_{\tilde{g}_{q}} \text {. }
$$

Given $q \in \partial M$ and $\psi_{q}^{\partial}: \mathbb{R}_{+}^{n} \rightarrow M$ the Fermi coordinates of $M$ defined in a neighborhood of $q$ we set

$$
\begin{aligned}
W_{\delta, q}(\xi) & :=U_{\delta}\left(\left(\psi_{q}^{\partial}\right)^{-1}(\xi)\right) \chi\left(\left(\psi_{q}^{\partial}\right)^{-1}(\xi)\right) \\
& =\frac{1}{\delta^{\frac{n-2}{2}}} U\left(\frac{y}{\delta}\right) \chi(y)=\frac{1}{\delta^{\frac{n-2}{2}}} U(x) \chi(\delta x)
\end{aligned}
$$

where $y=(z, t)$, with $z \in \mathbb{R}^{n-1}$ and $t \geq 0, \delta x=y=\left(\psi_{q}^{\partial}\right)^{-1}(\xi)$ and $\chi$ is a radial cut off function, with support in ball of radius $R, R$ being the injectivity radius for the Fermi coordinates.

Here $U_{\delta}(y)=\frac{1}{\delta^{\frac{n-2}{2}}} U\left(\frac{y}{\delta}\right)$ is the one parameter family of solution of the problem

$$
\left\{\begin{array}{cc}
-\Delta U_{\delta}=0 & \text { on } \mathbb{R}_{+}^{n} \\
\frac{\partial U_{\delta}}{\partial t}=-(n-2) U_{\delta}^{\frac{n}{n-2}} & \text { on } \partial \mathbb{R}_{+}^{n}
\end{array}\right.
$$

that is $U(z, t):=\frac{1}{\left[(1+t)^{2}+|z|^{2}\right]^{\frac{n-2}{2}}}$ is the standard bubble in $\mathbb{R}_{+}^{n}$.

Now, if we consider the linearized problem

$$
\left\{\begin{array}{cc}
-\Delta \phi=0 & \text { on } \mathbb{R}_{+}^{n}, \\
\frac{\partial \phi}{\partial t}+n U^{\frac{2}{n-2}} \phi=0 & \text { on } \partial \mathbb{R}_{+}^{n}, \\
\phi \in H^{1}\left(\mathbb{R}_{+}^{n}\right) . &
\end{array}\right.
$$

we have that every solution of (17) is a linear combination of the functions $j_{1}, \ldots, j_{n}$ defined by

$$
j_{i}=\frac{\partial U}{\partial y_{i}}, i=1, \ldots n-1 ; \quad j_{n}=\frac{n-2}{2} U+\sum_{a=1}^{n} y_{a} \frac{\partial U}{\partial y_{a}}
$$

(for a proof of this result, see, for instance, [16. Lemma 6]). By means of functions $j_{i}$ we define we define, for $b=1, \ldots, n$

$$
Z_{\delta, q}^{b}(\xi)=\frac{1}{\delta^{\frac{n-2}{2}}} j_{b}\left(\frac{1}{\delta}\left(\psi_{q}^{\partial}\right)^{-1}(\xi)\right) \chi\left(\left(\psi_{q}^{\partial}\right)^{-1}(\xi)\right)
$$

and we decompose $H^{1}(M)$ in the direct sum of the following two subspaces

$$
\begin{aligned}
& \tilde{K}_{\delta, q}=\operatorname{Span}\left\langle\Lambda_{q} Z_{\delta, q}^{1}, \ldots, \Lambda_{q} Z_{\delta, q}^{n}\right\rangle \\
& \tilde{K}_{\delta, q}^{\perp}=\left\{\varphi \in H^{1}(M):\left\langle\left\langle\varphi, \Lambda_{q} Z_{\delta, q}^{b}\right\rangle\right\rangle_{g}=0, b=1, \ldots, n\right\}
\end{aligned}
$$

and we define the projections

$$
\tilde{\Pi}=H^{1}(M) \rightarrow \tilde{K}_{\delta, q} \text { and } \tilde{\Pi}^{\perp}=H^{1}(M) \rightarrow \tilde{K}_{\delta, q}^{\perp} .
$$

Given $q \in \partial M$ we also define in a similar way

$$
V_{\delta, q}(\xi)=\frac{1}{\delta^{\frac{n-2}{2}}} v_{q}\left(\frac{1}{\delta}\left(\psi_{q}^{\partial}\right)^{-1}(\xi)\right) \chi\left(\left(\psi_{q}^{\partial}\right)^{-1}(\xi)\right)
$$


and

$$
\left(v_{q}\right)_{\delta}(y)=\frac{1}{\delta^{\frac{n-2}{2}}} v_{q}\left(\frac{y}{\delta}\right)
$$

here $v_{q}: \mathbb{R}_{+}^{n} \rightarrow \mathbb{R}$ is the solution of the linear problem

$$
\left\{\begin{array}{cc}
-\Delta v=\left[\frac{1}{3} \bar{R}_{i j k l}(q) y_{k} y_{l}+R_{n i n j}(q) y_{n}^{2}\right] \partial_{i j}^{2} U & \text { on } \mathbb{R}_{+}^{n} \\
\frac{\partial v}{\partial y_{n}}=-n U^{\frac{2}{n-2}} v & \text { on } \partial \mathbb{R}_{+}^{n}
\end{array}\right.
$$

These solutions will be used in the blow up estimate in the next: indeed, by means of the choice of $v_{q}$ we will be able to cancel the first order term in the following formula (31) and to have the correct size of the remainder term in the finite dimensional reduction (Lemma 4).

Lemma 3. There exists a unique $v_{q}: \mathbb{R}_{+}^{n} \rightarrow \mathbb{R}$ solution of the problem (19) $L^{1}\left(\mathbb{R}_{+}^{n}\right)$ ortogonal to $j_{b}$ for all $b=1, \ldots, n$. Moreover the function $q \mapsto v_{q}$ is $C^{2}(\partial M)$ and it holds

$$
\begin{gathered}
\left|\nabla^{\tau} v_{q}(y)\right| \leq C(1+|y|)^{4-\tau-n} \text { for } \tau=0,1,2, \\
\int_{\partial \mathbb{R}_{+}^{n}} U^{\frac{n}{n-2}}(t, z) v_{q}(t, z) d z=0
\end{gathered}
$$

and

$$
\int_{\partial \mathbb{R}_{+}^{n}} v_{q}(t, z) \Delta v_{q}(t, z) d z \leq 0
$$

where $y \in \mathbb{R}_{+}^{n}, y=(t, z)$ with $t \geq 0$ and $z \in \mathbb{R}^{n-1}$.

The proof of this result is postponed to Appendix.

We have the well know maps:

$$
\begin{aligned}
& i_{g}: H^{1}(M) \rightarrow L^{t}(\partial M) \\
& i_{g}^{*}: L^{t^{\prime}}(\partial M) \rightarrow H^{1}(M)
\end{aligned}
$$

for $1 \leq t \leq \frac{2(n-1)}{n-2}$ (and for $1 \leq t<\frac{2(n-1)}{n-2}$ the embedding $i$ is compact).

Given $f \in L^{\frac{2(n-1)}{n-2}}(\partial M)$ there exists a unique $v \in H^{1}(M)$ such that

$$
\begin{aligned}
v=i_{g}^{*}(f) & \Longleftrightarrow\langle\langle v, \varphi\rangle\rangle_{g}=\int_{\partial M} f \varphi d \sigma \text { for all } \varphi \\
& \Longleftrightarrow\left\{\begin{array}{cr}
-\Delta_{g} v+\frac{n-2}{4(n-1)} R_{g} v=0 & \text { on } M ; \\
\frac{\partial v}{\partial \nu}+\frac{n-2}{2} h_{g} v=f & \text { on } \partial M .
\end{array}\right.
\end{aligned}
$$

The functional defined on $H^{1}(M)$ associated to (7) is

$$
\begin{aligned}
J_{\varepsilon, g}(v): & =\frac{1}{2} \int_{M}\left|\nabla_{g} v\right|^{2}+\frac{n-2}{4(n-1)} R_{g} v^{2} d \mu_{g}+\frac{n-2}{4} \int_{\partial M} h_{g} v^{2} d \sigma_{g} \\
& +\frac{1}{2} \int_{\partial M} \varepsilon \gamma v^{2} d \sigma_{g}-\frac{(n-2)^{2}}{2(n-1)} \int_{\partial M}\left(v^{+}\right)^{\frac{2(n-1)}{n-2}} d \sigma_{g} .
\end{aligned}
$$

Notice that, if we define

$$
\begin{aligned}
\tilde{J}_{\varepsilon, \tilde{g}_{q}}(u): & =\frac{1}{2} \int_{M}\left|\nabla_{\tilde{g}_{q}} u\right|^{2}+\frac{n-2}{4(n-1)} R_{\tilde{g}_{q}} u^{2} d \mu_{\tilde{g}_{q}}+\frac{n-2}{4} \int_{\partial M} h_{\tilde{g}_{q}} u^{2} d \sigma_{\tilde{g}_{q}} \\
& +\frac{1}{2} \int_{\partial M} \varepsilon \tilde{\gamma} u^{2} d \sigma_{\tilde{g}_{q}}-\frac{(n-2)^{2}}{2(n-1)} \int_{\partial M}\left(u^{+}\right)^{\frac{2(n-1)}{n-2}} d \sigma_{v}
\end{aligned}
$$

then we have

$$
J_{\varepsilon, g}\left(\Lambda_{q} u\right)=\tilde{J}_{\varepsilon, \tilde{g}_{q}}(u)
$$




\section{The Finite DIMENSIONAL REDUCTION}

Solving problem (7) is equivalent to find $v \in H^{1}(M)$ such that

$$
v=i_{g}^{*}(f(v)-\varepsilon \gamma v)
$$

where

$$
f(v)=(n-2)\left(v^{+}\right)^{\frac{n}{n-2}}
$$

We remark that, if $v \in H_{g}^{1}(M)$, then $f(v) \in L^{\frac{2(n-1)}{n}}(\partial M)$.

We look for a positive solution of (7) in the form

$$
v=\Lambda_{q} u=\tilde{W}_{\delta, q}+\delta^{2} \tilde{V}_{\delta, q}+\tilde{\Phi}
$$

(we recall that, if $f: M \rightarrow \mathbb{R}$, we use the notation $\tilde{f}:=\Lambda_{q} f$ ). Thus we can rewrite, in light of the previous orthogonal decomposition, Problem (7) as

$$
\tilde{\Pi}\left\{\tilde{W}_{\delta, q}+\delta^{2} \tilde{V}_{\delta, q}+\tilde{\Phi}-i_{g}^{*}\left[f\left(\tilde{W}_{\delta, q}+\delta^{2} \tilde{V}_{\delta, q}+\tilde{\Phi}\right)-\varepsilon \gamma \tilde{W}_{\delta, q}+\delta^{2} \tilde{V}_{\delta, q}+\tilde{\Phi}\right]\right\}=0
$$

$$
\tilde{\Pi}^{\perp}\left\{\tilde{W}_{\delta, q}+\delta^{2} \tilde{V}_{\delta, q}+\tilde{\Phi}-i_{g}^{*}\left[f\left(\tilde{W}_{\delta, q}+\delta^{2} \tilde{V}_{\delta, q}+\tilde{\Phi}\right)-\varepsilon \gamma \tilde{W}_{\delta, q}+\delta^{2} \tilde{V}_{\delta, q}+\tilde{\Phi}\right]\right\}=0 .
$$

Now we define the linear operator $L: \tilde{K}_{\delta, q}^{\perp} \rightarrow \tilde{K}_{\delta, q}^{\perp}$ as

$$
L(\tilde{\Phi})=\tilde{\Pi}^{\perp}\left\{\tilde{\Phi}-i_{g}^{*}\left(f^{\prime}\left(\tilde{W}_{\delta, q}+\delta^{2} \tilde{V}_{\delta, q}\right)[\tilde{\Phi}]\right)\right\}
$$

we define a nonlinear term $N(\tilde{\Phi})$ and a remainder term $\mathrm{R}$ as

$$
N(\tilde{\Phi})=\tilde{\Pi}^{\perp}\left\{i_{g}^{*}\left(f\left(\tilde{W}_{\delta, q}+\delta^{2} \tilde{V}_{\delta, q}+\tilde{\Phi}\right)-f\left(\tilde{W}_{\delta, q}+\delta^{2} \tilde{V}_{\delta, q}\right)-f^{\prime}\left(\tilde{W}_{\delta, q}+\delta^{2} \tilde{V}_{\delta, q}\right)[\tilde{\Phi}]\right)\right\}
$$

$$
R=\tilde{\Pi}^{\perp}\left\{i_{g}^{*}\left(f\left(\tilde{W}_{\delta, q}+\delta^{2} \tilde{V}_{\delta, q}\right)\right)-\tilde{W}_{\delta, q}-\delta^{2} \tilde{V}_{\delta, q}\right\}
$$

so equation (26) becomes

$$
L(\tilde{\Phi})=N(\tilde{\Phi})+R-\tilde{\Pi}^{\perp}\left\{i_{g}^{*}\left(\varepsilon \gamma\left(\tilde{W}_{\delta, q}+\delta^{2} \tilde{V}_{\delta, q}+\tilde{\Phi}\right)\right)\right\}
$$

Lemma 4. Assume $n \geq 10$, then it holds

$$
\|R\|_{g}=O\left(\varepsilon \delta+\delta^{3}\right)
$$

$C^{0}$-uniformly for $q \in \partial M$.

Proof. We recall that there is a unique $\Gamma$ such that

$$
\Gamma=i_{g}^{*}\left(f\left(\tilde{W}_{\delta, q}+\delta^{2} \tilde{V}_{\delta, q}\right)\right)
$$

that is, according to (23) equivalent to say that there exists a unique $\Gamma$ solving

$$
\left\{\begin{array}{cc}
-\Delta_{g} \Gamma+\frac{n-2}{4(n-1)} R_{g} \Gamma=0 & \text { on } M \\
\frac{\partial \Gamma}{\partial \nu}+\frac{n-2}{2} h_{g} \Gamma=(n-2)\left(\left(\tilde{W}_{\delta, q}+\delta^{2} \tilde{V}_{\delta, q}\right)^{+}\right)^{\frac{n}{n-2}} & \text { on } \partial M
\end{array}\right.
$$


By definition of $i_{g}^{*}$ we have that

$$
\begin{aligned}
\|R\|_{g}^{2}= & \left\|\Gamma-\tilde{W}_{\delta, q}-\delta^{2} \tilde{V}_{\delta, q}\right\|_{g}^{2} \\
= & \int_{M}-\left[L_{g}\left(\Gamma-\tilde{W}_{\delta, q}-\delta^{2} \tilde{V}_{\delta, q}\right)\right]\left(\Gamma-\tilde{W}_{\delta, q}-\delta^{2} \tilde{V}_{\delta, q}\right) d \mu_{g} \\
& +\int_{\partial M}\left[B_{g}\left(\Gamma-\tilde{W}_{\delta, q}-\delta^{2} \tilde{V}_{\delta, q}\right)\right]\left(\Gamma-\tilde{W}_{\delta, q}-\delta^{2} \tilde{V}_{\delta, q}\right) d \sigma_{g} \\
= & \int_{M}\left[-L_{g}\left(\tilde{W}_{\delta, q}+\delta^{2} \tilde{V}_{\delta, q}\right)\right] R d \mu_{g} \\
& +\int_{\partial M}\left[B_{g}(\Gamma)-B_{g}\left(\tilde{W}_{\delta, q}+\delta^{2} \tilde{V}_{\delta, q}\right)\right] R d \sigma_{g} \\
= & \int_{M}\left[-L_{\tilde{g}_{q}}\left(W_{\delta, q}+\delta^{2} V_{\delta, q}\right)\right] \Lambda_{q}^{-1} R d \mu_{\tilde{g}_{q}} \\
& +\int_{\partial M}\left[B_{\tilde{g}_{q}}\left(\Lambda_{q}^{-1} \Gamma\right)-B_{\tilde{g}_{q}}\left(W_{\delta, q}+\delta^{2} V_{\delta, q}\right)\right] \Lambda_{q}^{-1} R d \sigma_{\tilde{g}_{q}}
\end{aligned}
$$

We estimate

$$
\begin{aligned}
\int_{M}\left[\Delta_{\tilde{g}_{q}}\left(W_{\delta, q}+\delta^{2} V_{\delta, q}\right)\right] \Lambda_{q}^{-1} R d \mu_{\tilde{g}_{q}} & \leq\left\|\Delta_{\tilde{g}_{q}}\left(W_{\delta, q}+\delta^{2} V_{\delta, q}\right)\right\|_{L^{\frac{2 n}{n+2}}\left(M, \tilde{g}_{q}\right)}\left\|\Lambda_{q}^{-1} R\right\|_{\tilde{g}_{q}} \\
& \leq O\left(\delta^{3}\right)\|R\|_{g}
\end{aligned}
$$

In fact, recalling the expression fot Laplace Beltrami operator in local charts

$$
\begin{aligned}
\Delta_{\tilde{g}_{q}} & =\Delta_{\mathrm{euc}}+\left[\tilde{g}_{q}^{i j}(y)-\delta_{i j}\right] \partial_{i j}^{2} \\
& +\left[\partial_{i} \tilde{g}_{q}^{i j}(y)+\frac{\tilde{g}_{q}^{i j}(y) \partial_{i}\left|\tilde{g}_{q}\right|^{\frac{1}{2}}(y)}{\left|\tilde{g}_{q}\right|^{\frac{1}{2}}(y)}\right] \partial_{j}+\frac{\partial_{n}\left|\tilde{g}_{q}\right|^{\frac{1}{2}}(y)}{\left|\tilde{g}_{q}\right|^{\frac{1}{2}}(y)} \partial_{n}
\end{aligned}
$$

where $i, k=1, \ldots, n-1$ and $\Delta_{\text {euc }}$ is the euclidean Laplacian, by (9) and (11) and since $\Delta_{\text {euc }} U=0$, in variables $y=\delta x$ we have

$$
\begin{aligned}
\Delta_{\tilde{g}_{q}} W_{\delta, q} & =\frac{1}{\delta^{\frac{n-2}{2}}} \frac{1}{3}\left(\bar{R}_{i k j l} x_{k} x_{l}+R_{n i n j} x_{n}^{2}+\delta O\left(|x|^{3}\right)\right) \partial_{i j}^{2}(U(x) \chi(\delta x)) \\
& +\frac{1}{\delta^{\frac{n-2}{2}}} \frac{1}{3}\left(\bar{R}_{i i j l} x_{l}+\bar{R}_{i k j i} x_{k}+\delta O\left(|x|^{2}\right)\right) \partial_{j}(U(x) \chi(\delta x)) \\
& +\frac{1}{\delta^{\frac{n-2}{2}}} \delta^{2} O\left(|x|^{3}\right) \partial_{n}(U(x) \chi(\delta x))
\end{aligned}
$$

We remark that, by symmetry, $\bar{R}_{i i j l}=0$ and by [19, Prop $3.2(2)$ ] also $\bar{R}_{i k j i}=$ $-\bar{R}_{j k}=0$. Now, the definition of $v_{q}(19)$ is crucial to get

$$
\begin{aligned}
\delta^{2} \Delta_{\tilde{g}_{q}} V_{\delta, q} & =\frac{1}{\delta^{\frac{n-2}{2}}} \Delta_{\text {euc }}\left(v_{q}(x) \chi(\delta x)\right)+\frac{\delta^{2}}{\delta^{\frac{n-2}{2}}}\left(O\left(|x|^{2}\right) \partial_{i j} v_{q}+O(|x|) \partial_{j} v_{q}\right) \\
& =\frac{1}{\delta^{\frac{n-2}{2}}}\left(-\frac{1}{3}\left(\bar{R}_{i k j l} x_{k} x_{l}+R_{n i n j} x_{n}^{2}\right) \partial_{i j}^{2}(U(x) \chi(\delta x))+\delta O\left(|x|^{3}\right)\right)
\end{aligned}
$$

Thus, this term cancels the first order term in (30) and we get

$$
\left\|\Delta_{\tilde{g}_{q}}\left(W_{\delta, q}+\delta^{2} V_{\delta, q}\right)\right\|_{L^{\frac{2 n}{n+2}}(M)}=O\left(\delta^{n \frac{n+2}{2 n}} \frac{1}{\delta^{\frac{n-2}{2}}} \delta\right)=O\left(\delta^{3}\right)
$$

Also we have

$\int_{M} R_{\tilde{g}_{q}}\left(W_{\delta, q}+\delta^{2} V_{\delta, q}\right) \Lambda_{q}^{-1} R d \mu_{\tilde{g}} \leq c\left\|R_{\tilde{g}_{q}}\left(W_{\delta, q}+\delta^{2} V_{\delta, q}\right)\right\|_{L^{\frac{2 n}{n+2}}\left(M, \tilde{g}_{q}\right)}\left\|\Lambda_{q}^{-1} R\right\|_{L^{\frac{2 n}{n-2}}\left(M, \tilde{g}_{q}\right)}$

$$
\leq o\left(\delta^{3}\right)\|R\|_{g}
$$


In fact, since $R_{\tilde{g}_{q}}(q)=R_{\tilde{g}_{q}, i}(q)=R_{\tilde{g}_{q}, t}(q)=0$ (see [19]), by the decay of $U$ and since $\left|v_{q}(y)\right| \leq C|y|^{4-n}$ we have, in local coordinates

$$
\begin{array}{r}
\left\|R_{\tilde{g}_{q}}\left(W_{\delta, q}+\delta^{2} V_{\delta, q}\right)\right\|_{L^{\frac{2 n}{n+2}}\left(M, \tilde{g}_{q}\right)} \leq O\left(\delta^{2}\right)\left\{\int_{\mathbb{R}_{+}^{n}}\left[R_{\tilde{g}_{q}}(\delta y) U(y) \chi(\delta y)\right]^{\frac{2 n}{n+2}} d y\right\}^{\frac{n+2}{2 n}} \\
+O\left(\delta^{4}\right)\left\{\int_{\mathbb{R}_{+}^{n}}\left[R_{\tilde{g}_{q}}(\delta y) v_{q}(y) \chi(\delta y)\right]^{\frac{2 n}{n+2}} d y\right\}^{\frac{n+2}{2 n}} \\
=o\left(\delta^{3}\right)\left\{\int_{\mathbb{R}_{+}^{n}}\left[y^{2} U(y)\right]^{\frac{2 n}{n+2}} d y\right\}^{\frac{n+2}{2 n}}+O\left(\delta^{4}\right)\left\{\int_{\mathbb{R}_{+}^{n}}\left[v_{q}(y)\right]^{\frac{2 n}{n+2}} d y\right\}^{\frac{n+2}{2 n}}+o\left(\delta^{3}\right) \\
=o\left(\delta^{3}\right) \text { since } n>10 .
\end{array}
$$

For the boundary term we have

$$
\begin{aligned}
B_{\tilde{g}_{q}}\left(\Lambda_{q}^{-1} \Gamma\right)-B_{\tilde{g}_{q}}\left(W_{\delta, q}+\delta^{2} V_{\delta, q}\right) & \\
=\{(n-2) & \left.\left(\left(W_{\delta, q}+\delta^{2} V_{\delta, q}\right)^{+}\right)^{\frac{n}{n-2}}-(n-2)\left(W_{\delta, q}^{+}\right)^{\frac{n}{n-2}}-\delta^{2} \frac{\partial V_{\delta, q}}{\partial \nu}\right\} \\
& +\left\{(n-2)\left(W_{\delta, q}^{+}\right)^{\frac{n}{n-2}}-\frac{\partial W_{\delta, q}}{\partial \nu}\right\}-\frac{n-2}{2} h_{\tilde{g}}\left(W_{\delta, q}+\delta^{2} V_{\delta, q}\right)
\end{aligned}
$$

Since the boundary is umbilic, we have $h_{\tilde{g}_{q}}(q)=h_{\tilde{g}_{q}, i}(q)=h_{\tilde{g}_{q}, i k}(q)=0$ so we estimate

$$
\begin{aligned}
\int_{\partial M} h_{\tilde{g}_{q}}\left(W_{\delta, q}+\delta^{2} V_{\delta, q}\right) \Lambda_{q}^{-1} R d \sigma_{\tilde{g}_{q}} & \leq c\left\|h_{\tilde{g}_{q}}\left(W_{\delta, q}+\delta^{2} V_{\delta, q}\right)\right\|_{L^{\frac{2(n-1)}{n+2}}\left(M, \tilde{g}_{q}\right)}\left\|\Lambda_{q}^{-1} R\right\|_{\tilde{g}_{q}} \\
& =O\left(\delta^{4}\right)\|R\|_{g}
\end{aligned}
$$

Indeed, as in (32)

$$
\begin{gathered}
\left\|h_{\tilde{g}_{q}}\left(W_{\delta, q}+\delta^{2} V_{\delta, q}\right)\right\|_{L^{\frac{2(n-1)}{n+2}}\left(\partial M, \tilde{g}_{q}\right)} \leq\left\|h_{\tilde{g}_{q}} W_{\delta, q}\right\|_{L^{\frac{2(n-1)}{n+2}}\left(\partial M, \tilde{g}_{q}\right)}+\delta^{2}\left\|V_{\delta, q}\right\|_{L^{\frac{2(n-1)}{n+2}}\left(\partial M, \tilde{g}_{q}\right)} \\
=\left\{\int_{\mathbb{R}^{n-1}}\left[h_{\tilde{g}_{q}}(0, \delta z) U(0, z) \chi(0, \delta z)\right]^{\frac{2(n-1)}{n+2}} d z\right\}^{\frac{n+2}{2(n-1)}} \\
+\delta^{2}\left\{\int_{\mathbb{R}^{n-1}}\left[h_{\tilde{g}_{q}}(0, \delta z) v_{q}(0, z) \chi(0, \delta z)\right]^{\frac{2(n-1)}{n+2}} d z\right\}^{\frac{n+2}{2(n-1)}} \\
=o\left(\delta^{3}\right)\left\{\int_{\mathbb{R}^{n-1}}\left[z^{2} U(0, z)\right]^{\frac{2(n-1)}{n+2}} d z\right\}^{\frac{n+2}{2(n-1)}}+O\left(\delta^{4}\right)\left\|v_{q}(0, z)\right\|_{L^{\frac{2(n-1)}{n+2}}\left(\mathbb{R}^{n-1}\right)}+o\left(\delta^{3}\right) \\
=O\left(\delta^{4}\right) \text { since } n>10 .
\end{gathered}
$$

Here we considered $y=(t, z)$ with $t>0$ and $z \in \mathbb{R}^{n-1}$.

Easily we get

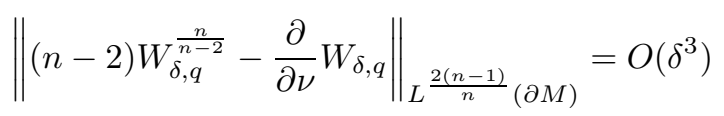

since $U$ solves (16). 
For the last term we estimate

$$
\begin{aligned}
& \int_{\partial M}\left\{(n-2)\left[\left(\left(W_{\delta, q}+\delta^{2} V_{\delta, q}\right)^{+}\right)^{\frac{n}{n-2}}-W_{\delta, q}^{\frac{n}{n-2}}\right]-\delta^{2} \frac{\partial V_{\delta, q}}{\partial \nu}\right\} \Lambda_{q}^{-1} R d \sigma_{\tilde{g}_{q}} \\
& \leq c\left\|(n-2)\left[\left(\left(W_{\delta, q}+\delta^{2} V_{\delta, q}\right)^{+}\right)^{\frac{n}{n-2}}-W_{\delta, q}^{\frac{n}{n-2}}\right]-\delta^{2} \frac{\partial V_{\delta, q}}{\partial \nu}\right\|_{L}{ }_{\frac{2(n-1)}{n}}\|R\|_{g}
\end{aligned}
$$

and, by Taylor expansion and by definition of the function $v_{q}$ (see (19))

$$
\begin{aligned}
& \left\|(n-2)\left[\left(\left(W_{\delta, q}+\delta^{2} V_{\delta, q}\right)^{+}\right)^{\frac{n}{n-2}}-W_{\delta, q}^{\frac{n}{n-2}}\right]-\delta^{2} \frac{\partial V_{\delta, q}}{\partial \nu}\right\|_{L \frac{2(n-1)}{n}\left(\partial M, \tilde{g}_{q}\right)} \\
& \leq\left\|(n-2)\left[\left(\left(U+\delta^{2} v_{q}\right)^{+}\right)^{\frac{n}{n-2}}-U^{\frac{n}{n-2}}\right]+\delta^{2} \frac{\partial v_{q}}{\partial t}\right\|_{L \frac{2(n-1)}{n}\left(\partial \mathbb{R}_{+}^{n}\right)}+o\left(\delta^{3}\right) \\
& \leq \delta^{2}\left\|n\left(\left(U+\theta \delta^{2} v_{q}\right)^{+}\right)^{\frac{2}{n-2}} v_{q}+\frac{\partial v_{q}}{\partial t}\right\|_{L \frac{2(n-1)}{n}\left(\partial \mathbb{R}_{+}^{n}\right)}+o\left(\delta^{3}\right) \\
& =\delta^{2} n\left\|\left(\left(U+\theta \delta^{2} v_{q}\right)^{+}\right)^{\frac{2}{n-2}} v_{q}-U^{\frac{2}{n-2}} v_{q}\right\|_{L \frac{2(n-1)}{n}\left(\partial \mathbb{R}_{+}^{n}\right)}+o\left(\delta^{3}\right) .
\end{aligned}
$$

We observe that, chosen a large positive $R$, we have $U+\theta \delta^{2} v_{q}>0$ in $B(0, R)$ for some $\delta$. Moreover, on the complementary of this ball, we have $\frac{c}{|y|^{n-2}} \leq U(y) \leq$ $\frac{C}{|y|^{n-2}}$ and $\left|v_{q}\right| \leq \frac{C_{1}}{|y|^{n-4}}$ for some positive constants $c, C, C_{1}$. So it is possible to prove that, for $\delta$ small enough, $U+\theta \delta^{2} v_{q}>0$ if $|y| \leq 1 / \delta$. At this point

$$
\begin{aligned}
\int_{\partial \mathbb{R}_{+}^{n}}\left[\left|\left(\left(U+\theta \delta^{2} v_{q}\right)^{+}\right)^{\frac{2}{n-2}}-U^{\frac{2}{n-2}}\right|\left|v_{q}\right|\right]^{\frac{2(n-1)}{n}} & {\left[\left|\left(\left(U+\theta \delta^{2} v_{q}\right)^{+}\right)^{\frac{2}{n-2}}-U^{\frac{2}{n-2}}\right|\left|v_{q}\right|\right]^{\frac{2(n-1)}{n}} d z } \\
& =\int_{U+\theta \delta v_{q}>0}\left[\left|\left(\left(U+\theta \delta^{2} v_{q}\right)^{+}\right)^{\frac{2}{n-2}}-U^{\frac{2}{n-2}}\right|\left|v_{q}\right|\right]^{\frac{2(n-1)}{n}} d z \\
& +\int_{U+\theta \delta v_{q} \leq 0}\left[\left.\left|\theta^{\frac{4(n-1)(n-4)}{n(n-2)}}\right| v_{q}\right|^{\frac{4(n-1)}{n}} d z\right. \\
& =\delta^{\frac{4(n-1)}{n}} \int_{U+\theta \delta v_{q}>0}\left(U+\theta_{1}^{2} v_{q}\right)^{\frac{4(n-1)}{n(n-2)}\left|v_{q}\right|^{\frac{2(n-1)}{n}} d z} \\
& \quad \int_{U+\theta \delta v_{q} \leq 0}\left(U+\theta_{1} \delta^{2} v_{q}\right)^{\frac{-2(n-1)(n-4)}{n(n-2)}}\left|v_{q}\right|^{\frac{4(n-1)}{n}} d z \\
& \int_{|z|>\frac{1}{\delta}} U^{\frac{4(n-1)}{n(n-2)}}\left|v_{q}\right|^{\frac{2(n-1)}{n}} d z
\end{aligned}
$$

and, since $n>10$ one can check that $\int_{U+\theta \delta v_{q}>0}\left(U+\theta_{1} \delta^{2} v_{q}\right)^{\frac{-2(n-1)(n-4)}{n(n-2)}}\left|v_{q}\right|^{\frac{4(n-1)}{n}} d z$ is bounded and that

$$
\begin{aligned}
\int_{|z|>\frac{1}{\delta}} U^{\frac{4(n-1)}{n(n-2)}}\left|v_{q}\right|^{\frac{2(n-1)}{n}} d z & \leq C \int_{|z|>\frac{1}{\delta}} \frac{1}{|z|^{\frac{4(n-1)}{n}}} \frac{1}{|z|^{\frac{2(n-1)(n-4)}{n}}} d z \\
& \leq C \int_{\frac{1}{\delta}}^{\infty} r^{-\frac{(n-2)^{2}}{n}}=O\left(\delta^{\frac{(n-2)^{2}-1}{n}}\right)=o\left(\delta^{\frac{4(n-1)}{n}}\right)
\end{aligned}
$$




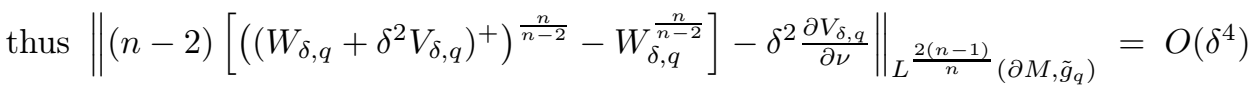
and

$$
\begin{array}{r}
\int_{\partial M}\left\{(n-2)\left[\left(\left(W_{\delta, q}+\delta^{2} V_{\delta, q}\right)^{+}\right)^{\frac{n}{n-2}}-W_{\delta, q}^{\frac{n}{n-2}}\right]-\delta \frac{\partial V_{\delta, q}}{\partial \nu}\right\} \Lambda_{q}^{-1} R d \sigma_{\tilde{g}_{q}} \\
\leq O\left(\delta^{4}\right)\|R\|_{g}
\end{array}
$$

At this point we can can use the same strategy of proposition 11 of [16] to prove the following result

Proposition 5. There exists a positive constant $C$ such that for $\varepsilon, \delta$ small, for any $q \in \partial M$ there exists a unique $\tilde{\Phi}=\tilde{\Phi}_{\varepsilon, \delta, q} \in \tilde{K}_{\delta, q}^{\perp}$ which solves (26) such that

$$
\|\tilde{\Phi}\|_{g}=\left\|\Lambda_{q} \Phi\right\|_{g} \leq C\left(\varepsilon \delta+\delta^{3}\right) .
$$

\section{The REDUCED FUnCtional}

In this section we perform the expansion of the functional with respect to the parameter $\varepsilon, \delta$.

Lemma 6. Assume $n \geq 10$. It holds

$$
J_{\varepsilon, g}\left(\tilde{W}_{\delta, q}+\delta^{2} \tilde{V}_{\delta, q}+\tilde{\Phi}\right)-J_{\varepsilon, g}\left(\tilde{W}_{\delta, q}+\delta^{2} \tilde{V}_{\delta, q}\right)=O\left(\left(\delta^{2}+\varepsilon \delta\right)\|\tilde{\Phi}\|_{g}+\|\tilde{\Phi}\|_{g}^{2}\right)
$$

$C^{0}$-uniformly for $q \in \partial M$.

The proof of this Lemma is postponed to the appendix.

We recall here an useful result contained in [19].

Remark 7. It holds

$$
\begin{aligned}
& I_{1}:=\int_{\mathbb{R}_{+}^{n}} \frac{t^{2}}{\left((1+t)^{2}+|z|^{2}\right)^{n-2}} d t d z=\frac{4(n-2)}{n+1} I_{2} \\
& I_{2}:=\int_{\mathbb{R}_{+}^{n}} \frac{t^{2}|z|^{4}}{\left((1+t)^{2}+|z|^{2}\right)^{n}} d t d z \\
& I_{3}:=\int_{\mathbb{R}_{+}^{n}} \frac{t^{4}|z|^{2}}{\left((1+t)^{2}+|z|^{2}\right)^{n}} d t d z=\frac{12}{(n-2)(n+1)} I_{2} \\
& I_{4}:=\int_{\mathbb{R}_{+}^{n}} \frac{|z|^{2}}{\left((1+t)^{2}+|z|^{2}\right)^{n-2}} d t d z
\end{aligned}
$$

and

$$
\int_{\mathbb{R}_{+}^{n}} \frac{t^{2} z_{i}^{4}}{\left((1+t)^{2}+|z|^{2}\right)^{n}} d t d z=3 \int_{\mathbb{R}_{+}^{n}} \frac{t^{2} z_{i}^{2} z_{j}^{2}}{\left((1+t)^{2}+|z|^{2}\right)^{n}} d t d z=\frac{3}{n^{2}-1} I_{2} .
$$

Lemma 8. It holds

$$
J_{\varepsilon, g}\left(\tilde{W}_{\delta, q}+\delta^{2} \tilde{V}_{\delta, q}\right)=A+\varepsilon \delta \gamma(q) B+\delta^{4} \varphi(q)+O\left(\varepsilon \delta^{3}\right)+O\left(\delta^{5}\right)
$$

where

$$
\varphi(q)=\frac{1}{2} \int_{\mathbb{R}_{+}^{n}} v_{q} \Delta v_{q} d t d z+\frac{(n-2)(n-8)}{4\left(n^{2}-1\right)} R_{n n, n n}(q) I_{2}-\frac{n-2}{96(n-1)}|\bar{W}(q)|^{2} I_{4},
$$

the constants $I_{2}, I_{4}$ are defined in Remark $\square$ and

$A=\frac{1}{2} \int_{\mathbb{R}_{+}^{n}}|\nabla U(t, z)|^{2} d t d z-\frac{(n-2)^{2}}{2(n-1)} \int_{\mathbb{R}^{n-1}} U(0, z)^{\frac{2(n-1)}{(n-2)}} d z, B=\frac{1}{2} \int_{\mathbb{R}^{n-1}} U(0, z)^{2} d z$ 
Here $\bar{W}(q)$ is the Weyl tensor restricted to boundary and we consider the local coordinates $y=(t, z)$ with $t>0$ and $z \in \mathbb{R}^{n-1}$.

Moreover

$$
\varphi(q) \leq 0 \text { for any } q \in \partial M
$$

Proof. First of all, we point out that the last claim (37) immediately follows by (22) and by the identity $R_{n n, n n}=-2 R_{n i n s}^{2}$ (see [19, Prop. $\left.3.2(7)\right]$ ).

Now, let us prove that (36) holds. We have

$$
\begin{aligned}
J_{\varepsilon, g}\left(\tilde{W}_{\delta, q}+\delta^{2} \tilde{V}_{\delta, q}\right)= & \frac{1}{2} \int_{M}\left|\nabla_{\tilde{g}_{q}}\left(W_{\delta, q}+\delta^{2} V_{\delta, q}\right)\right|^{2} d \mu_{\tilde{g}_{q}}+\frac{n-2}{8(n-1)} \int_{M} R_{\tilde{g}_{q}}\left(W_{\delta, q}+\delta^{2} V_{\delta, q}\right)^{2} d \mu_{\tilde{g}_{q}} \\
& +\frac{1}{2} \varepsilon \int_{\partial M} \Lambda_{q}^{-\frac{2}{n-2}} \gamma\left(W_{\delta, q}+\delta^{2} V_{\delta, q}\right)^{2} d \sigma_{\tilde{g}_{q}} \\
& -\frac{(n-2)^{2}}{2(n-1)} \int_{\partial M}\left[\left(\left(W_{\delta, q}+\delta^{2} V_{\delta, q}\right)^{+}\right)^{\frac{2(n-1)}{n-2}}-W_{\delta, q}^{\frac{2(n-1)}{n-2}}\right] d \sigma_{\tilde{g}_{q}} \\
& -\frac{(n-2)^{2}}{2(n-1)} \int_{\partial M} W_{\delta, q}^{\frac{2(n-1)}{n-2}} d \sigma_{\tilde{g}_{q}}+\frac{n-2}{4} \int_{\partial M} h_{\tilde{g}_{q}}\left(W_{\delta, q}+\delta^{2} V_{\delta, q}\right)^{2} d \sigma_{\tilde{g}_{q}} \\
= & A_{1}+A_{2}+A_{3}+A_{4}+A_{5}+A_{6} .
\end{aligned}
$$

We use the change of variables $y=\delta x=(\delta t, \delta z) \in \mathbb{R}_{+}^{n}$ with $t \geq 0$ and $z \in \mathbb{R}^{n-1}$.

On $\partial \mathbb{R}_{+}^{n}$ also we use $y=(0, \zeta)$

$$
\begin{aligned}
A_{6} & =\frac{n-2}{4} \int_{\partial M} h_{\tilde{g}_{q}}\left(W_{\delta, q}+\delta^{2} V_{\delta, q}\right)^{2} d \sigma_{\tilde{g}_{q}} \\
& =\frac{n-2}{4} \int_{\mathbb{R}^{n-1}} \frac{1}{\delta^{n-2}} h_{\tilde{g}_{q}}(0, \zeta)\left(U\left(0, \frac{\zeta}{\delta}\right)+\delta^{2} v_{q}\left(0, \frac{\zeta}{\delta}\right)\right)^{2} \chi^{2}(0, \zeta)\left|\tilde{g}_{q}\right|^{\frac{1}{2}}(0, \zeta) d y \\
& =\frac{n-2}{4} \delta \int_{\mathbb{R}^{n-1}} h_{\tilde{g}_{q}}(0, \delta z)\left(U(0, z)+\delta^{2} v_{q}(0, z)\right)^{2} \chi^{2}(0, \delta z)\left|\tilde{g}_{q}\right|^{\frac{1}{2}}(0, \delta z) d z .
\end{aligned}
$$

In light of (10), by Taylor expansion of $h_{\tilde{g}_{q}}(0, \delta z)$, since by symmetry the first term is zero and $n>10$, we have

$$
A_{6}=\frac{n-2}{4} \delta^{4} \int_{\mathbb{R}^{n-1}} \partial_{i j k} h_{\tilde{g}_{q}}(q) z_{i} z_{j} z_{k} U(0, z)^{2} d z+O\left(\delta^{5}\right)=O\left(\delta^{5}\right)
$$

In a similar way, expanding $R_{\tilde{g}_{q}}$ we get, by (12),

$$
\begin{aligned}
A_{2} & =\frac{n-2}{8(n-1)} \int_{\mathbb{R}_{+}^{n}} \delta^{2} R_{\tilde{g}_{q}}(\delta x)\left(U^{2}+\delta^{2} U v_{q}+\delta^{4} v_{q}^{2}\right) \chi^{2}(\delta x)\left|\tilde{g}_{q}\right|^{\frac{1}{2}}(\delta x) d x \\
& =\frac{n-2}{16(n-1)} \delta^{4} \int_{\mathbb{R}_{+}^{n}} \partial_{a b}^{2} R_{\tilde{g}_{q}}(q) x_{a} x_{b} U^{2} d x+O\left(\delta^{5}\right)
\end{aligned}
$$

By symmetry reasons, and recalling (12), we have

$A_{2}=\delta^{4} \frac{n-2}{16(n-1)}\left[\partial_{z_{i} z_{i}}^{2} R_{\tilde{g}_{q}}(q) \int_{\mathbb{R}_{+}^{n}} \frac{|z|^{2} U^{2}(z, t)}{n-1} d z d t+\partial_{t t}^{2} R_{\tilde{g}_{q}}(q) \int_{\mathbb{R}_{+}^{n}} t^{2} U^{2}(z, t) d z d t\right]+O\left(\delta^{5}\right)$

$$
\begin{aligned}
& =-\delta^{4} \frac{n-2}{96(n-1)^{2}}|\bar{W}(q)|^{2} \int_{\mathbb{R}_{+}^{n}}|z|^{2} U^{2}(z, t) d z d t \\
& +\delta^{4} \frac{n-2}{16(n-1)} \partial_{t t}^{2} R_{\tilde{g}_{q}} \int_{\mathbb{R}_{+}^{n}} t^{2} U^{2}(z, t) d z d t+O\left(\delta^{5}\right) .
\end{aligned}
$$


Analogously we have, since $\Lambda_{q}(q)=1$,

$$
\begin{aligned}
A_{3} & =\frac{1}{2} \varepsilon \delta \int_{\mathbb{R}^{n-1}} \Lambda_{q}^{-\frac{2}{n-2}} \gamma U^{2}(0, z) d z+O\left(\varepsilon \delta^{3}\right) \\
& =\frac{1}{2} \varepsilon \delta \gamma(q) \int_{\mathbb{R}^{n-1}} U^{2}(0, z) d z+O\left(\varepsilon \delta^{3}\right) .
\end{aligned}
$$

Also, by (9)

$$
\begin{aligned}
A_{5} & =-\frac{(n-2)^{2}}{2(n-1)} \int_{\mathbb{R}^{n-1}}[U(0, z) \chi(0, \delta z)]^{\frac{2(n-1)}{n-2}}\left|\tilde{g}_{q}(0, \delta z)\right|^{\frac{1}{2}} d z \\
& =-\frac{(n-2)^{2}}{2(n-1)} \int_{\mathbb{R}^{n-1}} U(0, z)^{\frac{2(n-1)}{n-2}} d z+O\left(\delta^{5}\right) .
\end{aligned}
$$

For $A_{4}$, expanding twice by Taylor formula, and since $\int_{\mathbb{R}^{n-1}} U(0, z)^{\frac{2}{n-2}} v_{q}(0, z) d z=$ 0 , we have

$$
\begin{aligned}
A_{4}= & -(n-2) \delta^{2} \int_{\mathbb{R}^{n-1}}\left[U(0, z)^{\frac{n}{n-2}} v_{q}\right] d z \\
& -\frac{n}{2} \delta^{4} \int_{\mathbb{R}^{n-1}}\left[\left(\left(U(0, z)+\theta \delta^{2} v_{q}\right)^{+}\right)^{\frac{2}{n-2}} v_{q}^{2}\right] d z+o\left(\delta^{4}\right) \\
= & -\frac{n}{2} \delta^{4} \int_{\mathbb{R}^{n-1}}\left[U(0, z)^{\frac{2}{n-2}} v_{q}^{2}\right] d z+o\left(\delta^{4}\right)
\end{aligned}
$$

Concerning the gradient term we have

$$
\begin{aligned}
A_{1} & =\frac{1}{2} \int_{M}\left|\nabla_{\tilde{g}_{q}} W_{\delta, q}\right|^{2} d \mu_{\tilde{g}_{q}}+\delta^{2} \int_{M} \nabla_{\tilde{g}_{q}} W_{\delta, q} \cdot \nabla V_{\delta, q} d \mu_{\tilde{g}_{q}}+\frac{\delta^{4}}{2} \int_{M}\left|\nabla_{\tilde{g}_{q}} V_{\delta, q}\right|^{2} d \mu_{\tilde{g}_{q}} \\
& =: L_{1}+L_{2}+L_{3} .
\end{aligned}
$$

We have, by (9) and (11) and integrating by parts

$$
\begin{aligned}
L_{3} & =\frac{\delta^{4}}{2} \int_{\mathbb{R}_{+}^{n}}\left[\tilde{g}_{q}^{i j}(\delta x) \partial_{y_{i}}\left(v_{q}(x) \chi(\delta x)\right) \partial_{x_{j}}\left(v_{q}(x) \chi(\delta x)\right)+\left(\partial_{x_{n}}\left(v_{q}(x) \chi(\delta x)\right)\right)^{2}\right]\left|\tilde{g}_{q}(\delta x)\right|^{\frac{1}{2}} d y \\
& =\frac{\delta^{4}}{2} \int_{\mathbb{R}_{+}^{n}}\left|\nabla v_{q}\right|^{2} d x+O\left(\delta^{5}\right) . \\
& =-\frac{\delta^{4}}{2} \int_{\mathbb{R}_{+}^{n}} v_{q} \Delta v_{q} d z d t+\frac{\delta^{4}}{2} \int_{\mathbb{R}^{n-1}} v_{q} \frac{\partial}{\partial \nu} v_{q} d z+O\left(\delta^{5}\right)
\end{aligned}
$$

$$
=-\frac{\delta^{4}}{2} \int_{\mathbb{R}_{+}^{n}} v_{q} \Delta v_{q} d z d t+\delta^{4} \frac{n}{2} \int_{\mathbb{R}^{n-1}} U^{\frac{2}{n-2}} v_{q}^{2} d z+O\left(\delta^{5}\right)
$$

In light of (16) and (21) we have

$$
\begin{aligned}
\int_{\mathbb{R}^{n}} \nabla U \nabla v_{q} d z d t & =-\int_{\mathbb{R}_{+}^{n}} \Delta U v_{q} d z d t+\int_{\mathbb{R}_{+}^{n-1}} \frac{\partial}{\partial \nu} U v_{q} d z d t \\
& =-(n-2) \int_{\mathbb{R}_{+}^{n-1}} U^{\frac{n}{n-2}} v_{q} d z d t=0
\end{aligned}
$$


So we have

$$
\begin{aligned}
L_{2}= & \delta^{2} \int_{\mathbb{R}_{+}^{n}} \nabla U \nabla v_{q} d z d t \\
& +\delta^{4} \int_{\mathbb{R}_{+}^{n}}\left(\frac{1}{3} \bar{R}_{i k j l} z_{k} z_{l} \partial_{z_{i}} U \partial_{z_{j}} v_{q}+R_{n i n j} t^{2} \partial_{z_{i}} U \partial_{z_{j}} v_{q}\right) d z d t+O\left(\delta^{5}\right) \\
& =\delta^{4} \int_{\mathbb{R}_{+}^{n}}\left(\frac{1}{3} \bar{R}_{i k j l} z_{k} z_{l} \partial_{z_{i}} U \partial_{z_{j}} v_{q}+R_{n i n j} t^{2} \partial_{z_{i}} U \partial_{z_{j}} v_{q}\right) d z d t+O\left(\delta^{5}\right)
\end{aligned}
$$

At this point we can calculate $A_{4}+L_{2}+L_{3}$. By (39), (40), (41) and by definition of $v_{q}$ (19) we get, integrating by parts

$$
\begin{aligned}
A_{4}+L_{2}+L_{3}= & -\frac{\delta^{4}}{2} \int_{\mathbb{R}_{+}^{n}} v_{q} \Delta v_{q} d z d t+\delta^{4} \int_{\mathbb{R}_{+}^{n}}\left(\frac{1}{3} \bar{R}_{i k j l} z_{k} z_{l}+R_{n i n j} t^{2}\right) \partial_{z_{i}} U \partial_{z_{j}} v_{q} d z d t+O\left(\delta^{5}\right) \\
= & -\frac{\delta^{4}}{2} \int_{\mathbb{R}_{+}^{n}} v_{q} \Delta v_{q} d z d t-\delta^{4} \int_{\mathbb{R}_{+}^{n}} \partial_{z_{j}}\left(\frac{1}{3} \bar{R}_{i k j l} z_{k} z_{l}+R_{n i n j} t^{2}\right) \partial_{z_{i}} U v_{q} d z d t \\
& -\delta^{4} \int_{\mathbb{R}_{+}^{n}}\left(\frac{1}{3} \bar{R}_{i k j l} z_{k} z_{l}+R_{n i n j} t^{2}\right) \partial_{z_{j}} \partial_{z_{i}} U v_{q} d z d t \\
& +\delta^{4} \int_{\partial \mathbb{R}_{+}^{n}}\left(\frac{1}{3} \bar{R}_{i k j l} z_{k} z_{l}+R_{n i n j} t^{2}\right) v_{q} \partial_{z_{i}} U \nu_{j} d z d t+O\left(\delta^{5}\right) \\
(42) \quad & \frac{\delta^{4}}{2} \int_{\mathbb{R}_{+}^{n}} v_{q} \Delta v_{q} d z d t+O\left(\delta^{5}\right)
\end{aligned}
$$

since $\nu_{j}=0$ for all $j=1, \ldots, n-1$, and by the symmetries of the curvature tensor and by (13) we have

$$
\begin{aligned}
\int_{\mathbb{R}_{+}^{n}} \partial_{z_{j}}\left(\frac{1}{3} \bar{R}_{i k j l} z_{k} z_{l}+\right. & \left.R_{n i n j} t^{2}\right) \partial_{z_{i}} U v_{q} d z d t \\
& =\frac{1}{3} \bar{R}_{i k j l} \int_{\mathbb{R}_{+}^{n}} \partial_{z_{j}}\left(z_{k} z_{l}\right) \partial_{z_{i}} U v_{q} d z d t \\
& =\frac{1}{3} \bar{R}_{i l} \int_{\mathbb{R}_{+}^{n}} z_{l} \partial_{z_{i}} U v_{q} d z d t+\frac{1}{3} \bar{R}_{i k j j} \int_{\mathbb{R}_{+}^{n}} z_{k} \partial_{z_{i}} U v_{q} d z d t=0 .
\end{aligned}
$$

Finally we have, by (9) and (11) and since the terms of odd degree disappear by symmetry

$$
\begin{aligned}
L_{1}= & \frac{1}{2} \int_{\mathbb{R}_{+}^{n}} \tilde{g}_{i j}(\delta x)\left(\partial_{x_{i}}(U \chi), \partial_{x_{j}}(U \chi)\right)+\partial_{x_{n}}(U \chi)\left|\tilde{g}_{q}\right|^{\frac{1}{2}} d x \\
& =\frac{1}{2} \int_{\mathbb{R}_{+}^{n}}|\nabla U|^{2} d z d t+\delta^{2} \int_{\mathbb{R}^{n}}\left(\frac{1}{3} \bar{R}_{i k j l} z_{k} z_{l}+R_{n i n j} t^{2}\right) \partial_{z_{i}} U \partial_{z_{j}} U d z d t \\
& +\frac{\delta^{4}}{2} \int_{\mathbb{R}_{+}^{n}}\left(\frac{1}{20} \bar{R}_{i k j l, m p}+\frac{1}{15} \bar{R}_{i k s l} \bar{R}_{j m s p}\right) z_{k} z_{l} z_{m} z_{p} \partial_{z_{i}} U \partial_{z_{j}} U d z d t \\
& +\frac{\delta^{4}}{2} \int_{\mathbb{R}_{+}^{n}}\left(\frac{1}{2} R_{n i n j, k l}+\frac{1}{3} \operatorname{Sym}_{i j}\left(\bar{R}_{i k s l} R_{n s n j}\right)\right) t^{2} z_{k} z_{l} \partial_{z_{i}} U \partial_{z_{j}} U d z d t \\
& +\frac{\delta^{4}}{2} \int_{\mathbb{R}_{+}^{n}}\left(\frac{1}{3} R_{n i n j, n k} t^{3} z_{k}+\frac{1}{12}\left(R_{n i n j, n n}+8 R_{n i n s} R_{n s n j}\right) t^{4}\right) \partial_{z_{i}} U \partial_{z_{j}} U d z d t \\
& +O\left(\delta^{5}\right) .
\end{aligned}
$$


Now we prove that all the terms of order $\delta^{2}$ vanish. Since $\partial_{z_{i}} U=(2-n) \frac{z_{i}}{\left((1+t)^{2}+|z|^{2}\right)^{\frac{n}{2}}}$ we have

$$
\begin{aligned}
& \int_{\mathbb{R}_{+}^{n}}\left(\frac{1}{3} \bar{R}_{i k j l} z_{k} z_{l}+R_{n i n j} t^{2}\right) \partial_{z_{i}} U \partial_{z_{j}} U d z d t \\
= & \frac{(n-2)^{2}}{3} \int_{\mathbb{R}_{+}^{n}} \bar{R}_{i k j l} \frac{z_{k} z_{l} z_{i} z_{j}}{\left((1+t)^{2}+|z|^{2}\right)^{n}} d z d t+(n-2)^{2} \int_{\mathbb{R}_{+}^{n}} R_{n i n j} \frac{t^{2} z_{i} z_{j}}{\left((1+t)^{2}+|z|^{2}\right)^{n}} d z d t .
\end{aligned}
$$

By symmetry reasons and by (13) we have

$$
\int_{\mathbb{R}_{+}^{n}} R_{n i n j} \frac{t^{2} z_{i} z_{j}}{\left((1+t)^{2}+|z|^{2}\right)^{n}} d z d t=\frac{1}{n-1} R_{n n} \int_{\mathbb{R}_{+}^{n}} \frac{t^{2}|z|^{2}}{\left((1+t)^{2}+|z|^{2}\right)^{n}} d z d t=0 .
$$

Moreover, by symmetry the integrals $\int_{\mathbb{R}^{n}} \frac{z_{k} z_{l} z_{i} z_{j}}{\left((1+t)^{2}+|z|^{2}\right)^{n}} d z d t$ are non zero only when $i=j=k=l, i=j \neq k=l, i=k \neq j=l$ and $i=l \neq j=k$. Since $\bar{R}_{i i j j}=0$ for all $i, j$ we get

$$
\begin{aligned}
& \int_{\mathbb{R}_{+}^{n}} \bar{R}_{i k j l} \frac{z_{k} z_{l} z_{i} z_{k}}{\left((1+t)^{2}+|z|^{2}\right)^{n}} d z d t \\
= & \sum_{i \neq k} \int_{\mathbb{R}_{+}^{n}} \bar{R}_{i k i k} \frac{z_{i}^{2} z_{k}^{2}}{\left((1+t)^{2}+|z|^{2}\right)^{n}} d z d t+\sum_{i \neq k} \int_{\mathbb{R}_{+}^{n}} \bar{R}_{i k k i} \frac{z_{i}^{2} z_{k}^{2}}{\left((1+t)^{2}+|z|^{2}\right)^{n}} d z d t=0 .
\end{aligned}
$$

By the symmetries of the curvature tensor (see [19, page 1614, formula C]) we get

$$
G_{1}:=\int_{\mathbb{R}_{+}^{n}}\left(\frac{1}{20} \bar{R}_{i k j l, m p}+\frac{1}{15} \bar{R}_{i k s l} \bar{R}_{j m s p}\right) z_{k} z_{l} z_{m} z_{p} \partial_{z_{i}} U \partial_{z_{j}} U d z d t=0
$$

Moreover, using that $R_{n n, n n}=-2 R_{n i n s}^{2}$, we get

$$
\begin{aligned}
G_{3} & :=\sum_{i} \frac{1}{12}\left(R_{n i n i, n n}+8 R_{n i n s} R_{n s n i}\right) \int_{\mathbb{R}_{+}^{n}} t^{4}\left|\partial_{z_{i}} U\right|^{2} d z d t \\
& =\frac{(n-2)^{2}}{12(n-1)}\left(R_{n n, n n}+8 R_{n i n s}^{2}\right) \int_{\mathbb{R}_{+}^{n}} \frac{t^{4}|z|^{2}}{\left((1+t)^{2}+|z|^{2}\right)^{n}} d z d t \\
& =\frac{n-2}{n^{2}-1}\left(R_{n n, n n}+8 R_{n i n s}^{2}\right) I_{2}=\frac{6(n-2)}{n^{2}-1} R_{n i n s}^{2} I_{2} .
\end{aligned}
$$

It remains

$$
G_{2}:=(n-2)^{2} \int_{\mathbb{R}_{+}^{n}}\left(\frac{1}{2} R_{n i n j, k l}+\frac{1}{3} \operatorname{Sym}_{i j}\left(\bar{R}_{i k s l} R_{n s n j}\right)\right) \frac{t^{2} z_{k} z_{l} z_{i} z_{j}}{\left((1+t)^{2}+|z|^{2}\right)^{n}} d z d t
$$

Again, by symmetry reasons, we have only to consider the cases $i=j=k=l$, $i=j \neq k=l, i=k \neq j=l$ and $i=l \neq j=k$. Then it is easy to see that the Symbol term gives no contribution. 
Finally, we have, by (35)

$$
\begin{aligned}
G_{2}= & \int_{\mathbb{R}^{n}} R_{n i n j, k l} \frac{t^{2} z_{k} z_{l} z_{i} z_{j}}{\left((1+t)^{2}+|z|^{2}\right)^{n}} d z d t=\sum_{i} R_{n i n i, i i} \int_{\mathbb{R}^{n}} \frac{t^{2} z_{1}^{4}}{\left((1+t)^{2}+|z|^{2}\right)^{n}} d z d t \\
+ & \left(\sum_{i \neq k} R_{n i n i, k k}+\sum_{i \neq j} R_{n i n j, i j}+\sum_{i \neq j} R_{n i n j, j i}\right) \int_{\mathbb{R}^{n}} \frac{t^{2} z_{1}^{2} z_{2}^{2}}{\left((1+t)^{2}+|z|^{2}\right)^{n}} d z d t \\
= & \left(3 \sum_{i} R_{n i n i, i i}+\sum_{i \neq k} R_{n i n i, k k}+\sum_{i \neq j} R_{n i n j, i j}+\sum_{i \neq j} R_{n i n j, j i}\right) \frac{1}{n^{2}-1} I_{2} \\
= & \left(\sum_{i, k} R_{n i n i, k k}+\sum_{i, j} R_{n i n j, i j}+\sum_{i, j} R_{n i n j, j i}\right) \frac{1}{n^{2}-1} I_{2}
\end{aligned}
$$

By [19, Proof of proposition 3.2, page 1609] we know $R_{n n, k k}=0$ for all $k=$ $1, \ldots, n-1$, so finally we have

$$
G_{2}=\frac{(n-2)^{2}}{n^{2}-1} I_{2} R_{n i n j, i j}
$$

Collecting all the terms, by (44), (45), (46), we have

$$
\begin{aligned}
L_{1}= & \frac{1}{2} \int_{\mathbb{R}^{n}}|\nabla U|^{2} d z d t+\frac{\delta^{4}}{2}\left(G_{1}+G_{2}+G_{3}\right) \\
= & \frac{1}{2} \int_{\mathbb{R}^{n}}|\nabla U|^{2} d z d t+ \\
& +\frac{\delta^{4}}{2} I_{2}\left(\frac{6(n-2)}{n^{2}-1} R_{n i n j}^{2}+\frac{(n-2)^{2}}{n^{2}-1} R_{n i n j, i j}\right)+O\left(\delta^{5}\right) .
\end{aligned}
$$

By (38) and (47), and by Remark 7 we have

$$
\begin{aligned}
A_{2}+L_{1}= & \frac{1}{2} \int_{\mathbb{R}^{n}}|\nabla U|^{2} d z d t-\delta^{4} \frac{n-2}{96(n-1)^{2}}|\bar{W}(q)|^{2} \int_{\mathbb{R}_{+}^{n}}|z|^{2} U^{2}(z, t) d z d t \\
& +\frac{\delta^{4}}{2} I_{2}\left(\frac{(n-2)^{2}}{2\left(n^{2}-1\right)} \partial_{t t}^{2} R_{\tilde{g}_{q}}+\frac{6(n-2)}{n^{2}-1} R_{n i n j}^{2}+\frac{(n-2)^{2}}{n^{2}-1} R_{n i n j, i j}\right) \\
= & \frac{1}{2} \int_{\mathbb{R}^{n}}|\nabla U|^{2} d z d t-\delta^{4} \frac{n-2}{96(n-1)^{2}}|\bar{W}(q)|^{2} \int_{\mathbb{R}_{+}^{n}}|z|^{2} U^{2}(z, t) d z d t \\
& +\frac{\delta^{4}(n-2)}{2\left(n^{2}-1\right)} I_{2}\left(\frac{(n-2)}{2} \partial_{t t}^{2} R_{\tilde{g}_{q}}+6 R_{n i n j}^{2}+(n-2) R_{n i n j, i j}\right)+o\left(\delta^{4}\right) \\
= & \frac{1}{2} \int_{\mathbb{R}^{n}}|\nabla U|^{2} d z d t-\delta^{4} \frac{n-2}{96(n-1)^{2}}|\bar{W}(q)|^{2} \int_{\mathbb{R}_{+}^{n}}|z|^{2} U^{2}(z, t) d z d t \\
& -\frac{\delta^{4}(n-2)(n-8)}{2\left(n^{2}-1\right)} I_{2} R_{n i n j}^{2}(q)+o\left(\delta^{4}\right) .
\end{aligned}
$$

In this computation we used the following formula [19, Formula (3.11) and Proposition $3.2(5)$ ]

This ends the proof.

$$
\partial_{t t}^{2} R_{\tilde{g}_{q}}=-2 R_{n i n j, i j}-2 R_{n i n j}^{2}
$$

\section{Proof of Theorem 1] completed}

First of all, we choose $\delta=\lambda \varepsilon^{\frac{1}{3}}$ with $\lambda \in[\alpha, \beta]$ compact subset of $(0,+\infty)$ (so that the second order term in the expansion of (36) have the same rate with respect 
to $\varepsilon$ ). Thus, summarizing the result of Section 3 we have that, for $\varepsilon$ small, for any $q \in \partial M$, for any $\lambda \in[\alpha, \beta]$, there exists a unique $\tilde{\Phi}=\tilde{\Phi}_{\varepsilon, \lambda, q} \in \tilde{K}_{\lambda \varepsilon}^{\perp}{ }_{\lambda{ }^{\frac{1}{3}}, q}$ which solves (26) such that

$$
\|\tilde{\Phi}\|_{g}=\left\|\Lambda_{q} \Phi\right\|_{g} \leq C \varepsilon
$$

Moreover

$$
\begin{array}{r}
J_{\varepsilon, g}\left(\tilde{W}_{\lambda \varepsilon^{\frac{1}{3}}, q}+\lambda^{2} \varepsilon^{\frac{2}{3}} \tilde{V}_{\lambda \varepsilon^{\frac{1}{3}}, q}+\tilde{\Phi}\right)=J_{\varepsilon, g}\left(\tilde{W}_{\lambda \varepsilon^{\frac{1}{3}}, q}+\lambda^{2} \varepsilon^{\frac{2}{3}} \tilde{V}_{\lambda \varepsilon^{\frac{1}{3}}, q}\right)+O\left(\varepsilon^{5 / 3}\right) \\
=A+\varepsilon^{\frac{4}{3}}\left[\lambda \gamma(q) B+\lambda^{4} \varphi(q)\right]+O\left(\varepsilon^{5 / 3}\right)
\end{array}
$$

$C^{0}$-uniformly for $q \in \partial M$ and $\lambda \in[\alpha, \beta]$, where $A, B, \varphi$ are defined in Lemma 8

Now, setting

$$
I_{\varepsilon}(\lambda, q):=J_{\varepsilon, g}\left(\tilde{W}_{\lambda \varepsilon^{\frac{1}{3}}, q}+\lambda^{2} \varepsilon^{\frac{2}{3}} \tilde{V}_{\lambda \varepsilon^{\frac{1}{3}}, q}+\tilde{\Phi}\right)
$$

and we can achieve the last part of our Theorem.

Lemma 9. If $(\bar{\lambda}, \bar{q}) \in(0,+\infty) \times \partial M$ is a critical point for the reduced functional $I_{\varepsilon}(\lambda, q)$, then the function $\tilde{W}_{\lambda \varepsilon^{\frac{1}{3}, q}}+\lambda^{2} \varepsilon^{\frac{2}{3}} \tilde{V}_{\lambda \varepsilon^{\frac{1}{3}, q}}+\tilde{\Phi}$ is a solution of (14).

Proof. Set $q=q(y)=\psi_{\bar{q}}^{\partial}(y)$. Since $(\bar{\lambda}, \bar{q})$ is a critical point for the $I_{\varepsilon}(\lambda, q)$ and since $\tilde{\Phi}$ is a solution of (26) we have, for $h=1, \ldots, n-1$, that there exists $c_{\varepsilon}^{a} \in \mathbb{R}$ such that

$$
\begin{aligned}
0= & \left.\frac{\partial}{\partial y_{h}} I_{\varepsilon}(\bar{\lambda}, q(y))\right|_{y=0} \\
= & \left.\left\langle\left\langle J_{\varepsilon, g}^{\prime}\left(\tilde{W}_{\bar{\lambda} \varepsilon^{\frac{1}{3}}, q(y)}+\bar{\lambda}^{2} \varepsilon^{\frac{2}{3}} \tilde{V}_{\bar{\lambda} \varepsilon^{\frac{1}{3}}, q(y)}+\tilde{\Phi}\right) \frac{\partial}{\partial y_{h}}\left(\tilde{W}_{\bar{\lambda}^{\frac{1}{3}}, q(y)}+\bar{\lambda}^{2} \varepsilon^{\frac{2}{3}} \tilde{V}_{\bar{\lambda} \varepsilon^{\frac{1}{3}}, q(y)}+\tilde{\Phi}\right)\right\rangle\right\rangle_{g}\right|_{y=0} \\
= & \left.\sum_{a=1}^{n} c_{\varepsilon}^{a}\left\langle\left\langle\Lambda_{q(y)} Z_{\bar{\lambda} \varepsilon^{\frac{1}{3}}, q(y)}^{a}, \frac{\partial}{\partial y_{h}}\left(\tilde{W}_{\bar{\lambda} \varepsilon^{\frac{1}{3}}, q(y)}+\bar{\lambda}^{2} \varepsilon^{\frac{2}{3}} \tilde{V}_{\bar{\lambda} \varepsilon^{\frac{1}{3}}, q(y)}+\tilde{\Phi}\right)\right\rangle\right\rangle_{g}\right|_{y=0} \\
= & \left.\sum_{a=1}^{n} c_{\varepsilon}^{a}\left\langle\left\langle\Lambda_{q(y)} Z_{\bar{\lambda} \varepsilon^{\frac{1}{3}}, q(y)}^{a}, \frac{\partial}{\partial y_{h}} \tilde{W}_{\bar{\lambda} \varepsilon^{\frac{1}{3}}, q(y)}\right\rangle\right\rangle_{g}\right|_{y=0} \\
& +\left.\varepsilon^{\frac{2}{3}} \bar{\lambda}^{2} \sum_{a=1}^{n} c_{\varepsilon}^{a}\left\langle\left\langle\Lambda_{q(y)} Z_{\bar{\lambda}_{\varepsilon} \frac{1}{3}, q(y)}^{a}, \frac{\partial}{\partial y_{h}} \tilde{V}_{\bar{\lambda} \varepsilon^{\frac{1}{3}}, q(y)}\right\rangle\right\rangle_{g}\right|_{y=0} \\
& \left.\sum_{a=1}^{n} c_{\varepsilon}^{a}\left\langle\left\langle\frac{\partial}{\partial y_{h}}\left(\Lambda_{q(y)} Z_{\bar{\lambda}_{\varepsilon} \frac{1}{3}, q(y)}^{a}\right), \tilde{\Phi}\right\rangle\right\rangle_{g}\right|_{y=0}
\end{aligned}
$$

using that

$$
\left\langle\left\langle\Lambda_{q(y)} Z_{\bar{\lambda} \varepsilon^{\frac{1}{3}}, q(y)}^{a}, \frac{\partial}{\partial y_{h}} \tilde{\Phi}\right\rangle\right\rangle_{g}=\left\langle\left\langle\frac{\partial}{\partial y_{h}}\left(\Lambda_{q(y)} Z_{\bar{\lambda} \varepsilon^{\frac{1}{3}}, q(y)}^{a}\right), \tilde{\Phi}\right\rangle\right\rangle_{g}
$$

since $\tilde{\Phi} \in K_{\bar{\lambda} \varepsilon^{\frac{1}{3}}, q(y)}^{\perp}$ for any $y$.

Arguing as in Lemma 6.1 and Lemma 6.2 of [21] we have

$$
\begin{aligned}
& \left\|\frac{\partial}{\partial y_{h}} Z_{\bar{\lambda} \varepsilon^{\frac{1}{3}}, q(y)}^{a}\right\|_{g}=O\left(\frac{1}{\overline{\bar{\lambda} \varepsilon^{\frac{1}{3}}}}\right) \quad\left\|\frac{\partial}{\partial y_{h}} W_{\bar{\lambda} \varepsilon^{\frac{1}{3}}, q(y)}\right\|_{g}=O\left(\frac{1}{\overline{\bar{\lambda} \varepsilon^{\frac{1}{3}}}}\right) \\
& \left\|\frac{\partial}{\partial y_{h}} V_{\bar{\lambda} \varepsilon^{\frac{1}{3}}, q(y)}\right\|_{g}=O\left(\frac{1}{\bar{\lambda} \varepsilon^{\frac{1}{3}}}\right) .
\end{aligned}
$$


For the first term we have

$$
\begin{aligned}
\left.\left\langle\left\langle\Lambda_{q(y)} Z_{\bar{\lambda} \varepsilon^{\frac{1}{3}}, q(y)}^{a}, \frac{\partial}{\partial y_{h}} \tilde{W}_{\bar{\lambda} \varepsilon^{\frac{1}{3}}, q}\right\rangle\right\rangle_{g}\right|_{y=0}= & \left.\left\langle\left\langle\Lambda_{q(y)} Z_{\bar{\lambda} \varepsilon^{\frac{1}{3}}, q(y)}^{a}, \Lambda_{q(y)} \frac{\partial}{\partial y_{h}} W_{\bar{\lambda} \varepsilon^{\frac{1}{3}}, q^{\prime}}\right\rangle\right\rangle_{g}\right|_{y=0} \\
& +\left.\left\langle\left\langle\Lambda_{q(y)} Z_{\bar{\lambda} \varepsilon^{\frac{1}{3}}, q(y)}^{a}, W_{\bar{\lambda} \varepsilon^{\frac{1}{3}}, q} \frac{\partial}{\partial y_{h}} \Lambda_{q}\right\rangle\right\rangle_{g}\right|_{y=0}
\end{aligned}
$$

so we get

$$
\begin{aligned}
\left\langle\left\langle\Lambda_{q(y)} Z_{\bar{\lambda} \varepsilon^{\frac{1}{3}}, q(y)}^{a}, \Lambda_{q(y)} \frac{\partial}{\partial y_{h}}\right.\right. & \left.\left.W_{\bar{\lambda} \varepsilon^{\frac{1}{3}}, q}\right\rangle\right\rangle\left._{g}\right|_{y=0} \\
& \left.=\frac{1}{\bar{\lambda} \varepsilon^{\frac{1}{3}}}\left\langle\left\langle\Lambda_{q} Z_{\bar{\lambda} \varepsilon^{\frac{1}{3}}, q}^{a}, Z_{\bar{\lambda} \varepsilon^{\frac{1}{3}}, q}^{h}\right)\right\rangle\right\rangle_{g}+o(1)=\frac{\delta_{i h}}{\bar{\lambda} \varepsilon^{\frac{1}{3}}}+o(1)
\end{aligned}
$$

And, by change of variables, that

$$
\left.\left\langle\left\langle\Lambda_{q(y)} Z_{\bar{\lambda} \varepsilon^{\frac{1}{3}}, q(y)}^{a}, W_{\bar{\lambda} \varepsilon^{\frac{1}{3}}, q} \frac{\partial}{\partial y_{h}} \Lambda_{q}\right\rangle\right\rangle_{g}\right|_{y=0}=O(1) .
$$

Similarly for the other terms we get

$$
\begin{aligned}
\left.\left\langle\left\langle\Lambda_{q(y)} Z_{\bar{\lambda} \varepsilon^{\frac{1}{3}}, q(y)}^{a}, \frac{\partial}{\partial y_{h}} \tilde{V}_{\bar{\lambda} \varepsilon^{\frac{1}{3}}, q(y)}\right\rangle\right\rangle_{g}\right|_{y=0} \leq\left\|\Lambda_{q(y)} Z_{\bar{\lambda} \varepsilon^{\frac{1}{3}}, q(y)}^{a}\right\|_{g}\left\|\frac{\partial}{\partial y_{h}} \tilde{V}_{\bar{\lambda} \varepsilon^{\frac{1}{3}}, q(y)}\right\|_{g}=O\left(\frac{1}{\bar{\lambda} \varepsilon^{\frac{1}{3}}}\right) \\
\left.\left\langle\left\langle\frac{\partial}{\partial y_{h}}\left(\Lambda_{q(y)} Z_{\bar{\lambda} \varepsilon^{\frac{1}{3}}, q(y)}^{a}\right), \tilde{\Phi}\right\rangle\right\rangle_{g}\right|_{y=0} \leq\left\|\Lambda_{q(y)} Z_{\bar{\lambda} \varepsilon^{\frac{1}{3}}, q(y)}^{a}\right\|_{g}\|\tilde{\Phi}\|_{g}=o(1) .
\end{aligned}
$$

So we conclude that

$$
0=\frac{1}{\lambda \varepsilon} \sum_{a=1}^{n} c_{\varepsilon}^{a}\left(\delta_{i h}+O(1)\right)
$$

which implies $c_{\varepsilon}^{a}=0$ for $a=1, \ldots, n$.

Analogously we proceed for $\left.\frac{\partial}{\partial \lambda} I_{\varepsilon}(\lambda, \bar{q})\right|_{\lambda=\bar{\lambda}}$, proving the claim.

For the sake of completeness, we recall the definition of $C^{0}$-stable critical point before proving Theorem 1 .

Definition 10. Let $f: \mathbb{R}^{n} \rightarrow \mathbb{R}$ be a $C^{1}$ function and let $K=\left\{\xi \in \mathbb{R}^{n}: \nabla f(\xi)=0\right\}$. We say that $\xi_{0} \in \mathbb{R}^{n}$ is a $C^{0}$-stable critical point if $\xi_{0} \in K$ and there exist $\Omega$ neighborhood of $\xi_{0}$ with $\partial \Omega \cap K=\emptyset$ and a $\eta>0$ such that for any $g: \mathbb{R}^{n} \rightarrow \mathbb{R}$ of class $C^{1}$ with $\|g-f\|_{C^{0}(\bar{\Omega})} \leq \eta$ we have a critical point of $g$ near $\Omega$.

We can complete now the proof of Theorem 1. By Lemma 9 and by the definition of $C^{0}$-stable critical point, we have to show that the function

$$
G(\lambda, q):=\left[\lambda \gamma(q) B+\lambda^{4} \varphi(q)\right]
$$

where $B$ and $\varphi$ are defined in Lemma 8 , admits a $C^{0}$-stable critical point. We know that $B>0$ by computation, and that $\gamma>0$ and $\varphi<0$ by the hypothesis of Th. 1 . Thus, one can check that there exists $0<\alpha<\beta$ such that any critical point $(\lambda, q) \in$ $(0,+\infty) \times \partial M$ of $G$ lies indeed in $(\alpha, \beta) \times \partial M$, because $\frac{\partial G}{\partial \lambda}=B \gamma(q)+4 \lambda^{3} \varphi(q)$ and $\frac{\partial G}{\partial \lambda}(\lambda, q)=0$ if and only if $\lambda^{3}=-\gamma(q) / \varphi(q)>0$.

Moreover for any number $L<0$ there exists $\bar{\lambda}>0$ such that $G(\lambda, q)<L$ for any $\lambda>\bar{\lambda}$ and $q \in \partial M$. Thus there exists a maximum point $\left(\lambda_{0}, q_{0}\right) \in(\alpha, \beta) \times \partial M$ which is $C^{0}$-stable, and we can conclude the proof.

Remark 11. We give another example of function $\gamma(q)$ such that problem (7) admits a positive solution. Let $q_{0} \in \partial M$ be a maximum point for $\varphi$. This point exists since $\partial M$ is compact. Now choose $\gamma \in C^{2}(\partial M)$ such that $\gamma$ has a positive local 
maximum in $q_{0}$. Then the pair $\left(\lambda_{0}, q_{0}\right)=\left(-\sqrt[3]{\frac{B \gamma\left(q_{0}\right)}{4 \varphi\left(q_{0}\right)}}, q_{0}\right)$ is a $C^{0}$-stable critical point for $G(\lambda, q)$.

In fact, we have

$$
\nabla_{\lambda, q} G=\left(B \gamma(q)+4 \lambda^{3} \varphi(q), \lambda B \nabla_{q} \gamma(q)+\lambda^{4} \nabla_{q} \varphi(q)\right)
$$

which vanishes for $\left(\lambda_{0}, q_{0}\right)=\left(-\sqrt[3]{\frac{B \gamma\left(q_{0}\right)}{4 \varphi\left(q_{0}\right)}}, q_{0}\right)$. Moreover the Hessian matrix is

$$
G_{\lambda, q}^{\prime \prime}\left(\lambda_{0}, q_{0}\right)=\left(\begin{array}{cc}
2 \varphi\left(q_{0}\right) & 0 \\
0 & \lambda_{0} \gamma_{q}^{\prime \prime}\left(q_{0}\right)+\lambda_{0}^{4} \varphi_{q}^{\prime \prime}\left(q_{0}\right)
\end{array}\right)
$$

which is negative definite. Thus $\left(\lambda_{0}, q_{0}\right)=\left(-\sqrt[3]{\frac{B \gamma\left(q_{0}\right)}{4 \varphi\left(q_{0}\right)}}, q_{0}\right)$ is a maximum, $C^{0}$ stable, point for $G(\lambda, q)$.

\section{Appendix}

Here we collect the proofs of the technical lemmas we claimed before.

Proof of Lemma 3. We follow the strategy of [1, Prop 5.1]. To prove the existence of a solution of (19) we have to show that the given term $\left[\frac{1}{3} \bar{R}_{i j k l}(q) z_{k} z_{l}+R_{n i n j}(q) t^{2}\right] \partial_{i j}^{2} U$ is $L_{2}\left(\partial \mathbb{R}_{+}^{n}\right)$-orthogonal to the functions $j_{1}, \ldots, j_{n}$. For $l=1, \ldots, n-1$ we have

$$
\begin{aligned}
\int_{\mathbb{R}_{+}^{n}}\left[\frac{1}{3} \bar{R}_{i j k l}(q) z_{k} z_{l}+R_{n i n j}(q) t^{2}\right] \partial_{i j}^{2} U j_{b} \\
=\int_{\mathbb{R}_{+}^{n}}\left[\frac{1}{3} \bar{R}_{i j k l}(q) z_{k} z_{l}+R_{n i n j}(q) t^{2}\right] \partial_{i j}^{2} U \partial_{l} U d z d t=0
\end{aligned}
$$

by symmetry, since the integrand is odd with respect to the $z$ variables.

For the last term, since when $i \neq j$ we have

$$
\partial_{i j} U=\frac{n(n-2) z_{i} z_{j}}{\left((1+t)^{2}+|z|^{2}\right)^{\frac{n+2}{2}}}
$$

and since when $i=j$ we have $\bar{R}_{i i k l}=0$ and, by (13), $R_{n i n i}=R_{n n}=0$ we have

$$
\begin{aligned}
\int_{\mathbb{R}_{+}^{n}}\left[\frac{1}{3} \bar{R}_{i j k l}(q) z_{k} z_{l}+R_{n i n j}(q) t^{2}\right] & \partial_{i j}^{2} U U d z d t \\
& =\sum_{i \neq j} \sum_{k} \int_{\mathbb{R}_{+}^{n}}\left[\frac{1}{3} \bar{R}_{i j k l}(q) z_{k} z_{l}+R_{n i n j}(q) t^{2}\right] \frac{n(n-2) z_{i} z_{j}}{\left((1+t)^{2}+|z|^{2}\right)^{n}}
\end{aligned}
$$

and since $i \neq j$, by symmetry all the terms containing $t^{2} z_{i} z_{j}$ vanish and the others terms are non zero only when $i=k$ and $j=l$ or when $j=k$ and $i=l$, thus

$$
\begin{gathered}
\int_{\mathbb{R}_{+}^{n}}\left[\frac{1}{3} \bar{R}_{i j k l}(q) z_{k} z_{l}+R_{n i n j}(q) t^{2}\right] \partial_{i j}^{2} U U d z d t \\
=\sum_{k} \int_{\mathbb{R}_{+}^{n}}\left[\frac{1}{3} \bar{R}_{k l k l}(q)+\frac{1}{3} \bar{R}_{l k k l}(q)\right] \frac{n(n-2) z_{k}^{2} z_{l}^{2}}{\left((1+t)^{2}+|z|^{2}\right)^{-n}}=0
\end{gathered}
$$


since $\bar{R}_{k l k l}(q)=-\bar{R}_{l k k l}(q)$. Moreover

$$
\begin{aligned}
\int_{\mathbb{R}_{+}^{n}} & {\left[\frac{1}{3} \bar{R}_{i j k l}(q) z_{k} z_{l}+R_{n i n j}(q) t^{2}\right] \partial_{i j}^{2} U y_{b} \partial_{b} U d t z } \\
= & n(2-n) \sum_{i \neq j} \sum_{k, s} \int_{\mathbb{R}_{+}^{n}}\left[\frac{1}{3} \bar{R}_{i j k l}(q) z_{k} z_{l}+R_{n i n j}(q) t^{2}\right] \frac{z_{i} z_{j}\left(z_{s} z_{s}+t(1+t)\right)}{\left((1+t)^{2}+|z|^{2}\right)^{-n-1}} \\
= & n(2-n) \sum_{k} \int_{\mathbb{R}_{+}^{n}}\left[\frac{1}{3} \bar{R}_{k l k l}(q)+\frac{1}{3} \bar{R}_{l k k l}(q)\right] \frac{z_{k}^{2} z_{l}^{2}\left(\sum_{s} z_{s} z_{s}+t(1+t)\right)}{\left((1+t)^{2}+|z|^{2}\right)^{-n-1}}=0 .
\end{aligned}
$$

Then there exists a solution. Also there exists a unique solution $v_{q}$ which is $L_{2}\left(\partial \mathbb{R}_{+}^{n}\right)$-orthogonal to $j_{b}$ for $b=1, \cdots, n$.

To prove the estimates (21) and (22) we use the inversion $F: \mathbb{R}_{+}^{n} \rightarrow B^{n}$ $\{(0, \ldots, 0-1)\}$ where $B^{n} \subset \mathbb{R}^{n}$ is the closed ball centered in $(0, \ldots, 0,-1 / 2)$ and radius $1 / 2$. The explicit expression for $F$ is

$$
F\left(y_{1}, \ldots, y_{n}\right)=\frac{\left(y_{1}, \ldots, y_{n-1}, y_{n}+1\right)}{y_{1}^{2}+\cdots+y_{n-1}^{2}+\left(y_{n}+1\right)^{2}}+(0, \ldots, 0-1) .
$$

We set

$$
f_{q}(F(y))=\left[\frac{1}{3} \bar{R}_{i j k l}(q) y_{k} y_{l}+R_{n i n j}(q) y_{n}^{2}\right] \partial_{i j}^{2} U(y) U^{-\frac{n+2}{n-2}}(y) .
$$

By direct computation we have $\left|f_{i}(F(y))\right| \leq C(1+|y|)^{4}$, so we have

$$
\left|f_{q}(\xi)\right| \leq C\left(1+\frac{1}{|\xi|}\right)^{4} \leq C \frac{1}{(1+|\xi|)^{4}}
$$

So it is possible to smoothly extend $f_{q}$ to the whole $B^{n}$, and it turns out that if $v_{q}$ solves (19), then $\bar{v}_{q}:=\left(U^{-1} v_{q}\right) \circ F^{-1}$ solves

$$
\left\{\begin{array}{cc}
-\Delta \bar{v}=f_{q} & \text { on } B^{n} \\
\frac{\partial v}{\partial y_{n}}+2 \bar{v}=0 & \text { on } \partial B^{n}
\end{array} .\right.
$$

Then existence and uniqueness of $\bar{v}_{q}$ are standard. To prove the decadence estimates, fixed $w \in B^{n}$, consider the Green's function $G(\xi, w)$ with boundary condition $\left(\frac{\partial}{\partial \nu}+2\right) G=0$. Then by Green's formula and by (49) we have

$$
\bar{v}_{q}(\xi)=\int_{B^{n}} G(\xi, w) \Delta \bar{v}_{q}(\xi)+\int_{\partial B^{n}} \bar{v}_{q} \frac{\partial}{\partial \nu} G-G \frac{\partial}{\partial \nu} \bar{v}_{q}=-\int_{B^{n}} G(\xi, w) f_{q}(\xi)
$$

and, in light of (48) we have

$$
\left|\bar{v}_{q}(\xi)\right| \leq C \int_{B^{n}}|\xi-w|^{2-n}(1+|\xi|)^{-4}
$$

and by [5, Prop 4.12 page 108] that $\left|\bar{v}_{q}(\xi)\right| \leq C(1+|\xi|)^{-2}$ and by the definition of $\bar{v}_{q}$ we deduce

$$
\left|v_{q}(y)\right| \leq C(1+|y|)^{4-n} .
$$

The estimates on the first and the second derivatives of $v_{q}$ can be achieved in a similar way.

It remains to prove (21) and (22). Notice that, changing of variables and proceeding as at the beginning of this proof, we have

$$
\int_{B^{n}} f_{q}(\xi) d \xi=\int_{\mathbb{R}_{+}^{n}} \frac{\left[\frac{1}{3} \bar{R}_{i j k l}(q) y_{k} y_{l}+R_{n i n j}(q) y_{n}^{2}\right] \partial_{i j}^{2} U(y) U^{-\frac{n+2}{n-2}}(y)}{\left(y_{1}^{2}+\cdots+y_{n-1}^{2}+\left(y_{n}+1\right)^{2}\right)^{n}} d y=0 .
$$

So we have, using (49) and integrating by parts, that

$$
0=\int_{B^{n}} f_{q}=-\int_{B^{n}} \Delta \bar{v}_{q}=-\int_{\partial B^{n}} \frac{\partial}{\partial \nu} \bar{v}_{q}=-\int_{\partial B^{n}} 2 \bar{v}_{q}
$$


and, changing variables again,

$$
\begin{aligned}
0=\int_{\partial B^{n}} 2 \bar{v}_{q}(\xi) d \xi_{1} \ldots d \xi_{n-1}=\int_{\partial \mathbb{R}_{+}^{n}} U^{-1}(y) & v_{q}(y) U^{\frac{2(n-1)}{n-2}}(y) d y_{1} \ldots d y_{n-1} \\
& =\int_{\partial \mathbb{R}_{+}^{n}} U^{\frac{n}{n-2}}(y) v_{q}(y) d y_{1} \ldots d y_{n-1} .
\end{aligned}
$$

It is known (see [1]), that it holds, on $H^{1}\left(B^{n}\right)$,

$$
\inf _{\int_{\partial B_{n}} \phi=0} \frac{\int_{B^{n}}|\nabla \phi|^{2}}{\int_{\partial B^{n}}|\phi|^{2}}=2 .
$$

Since, by (50), we know that $\int_{\partial B^{n}} \bar{v}_{q}=0$, we get

$$
2 \int_{\partial B^{n}} \bar{v}_{q}^{2} \leq \int_{B^{n}}\left|\nabla \bar{v}_{q}\right|^{2}
$$

so, integrating by parts

$$
-\int_{B^{n}} \bar{v}_{q} \Delta \bar{v}_{q}=\int_{B^{n}}\left|\nabla \bar{v}_{q}\right|^{2}-2 \int_{\partial B^{n}} \bar{v}_{q}^{2} \geq 0 .
$$

By the properties of the inversion $F$ (see [1, formula (5.10)]) we have also

$$
-\int_{B^{n}} \bar{v}_{q} \Delta \bar{v}_{q}=-\int_{\mathbb{R}_{+}^{n}} v_{q} \Delta v_{q}
$$

Finally, we want to prove that $v_{q} \in C^{2}(\partial M)$. Let $q_{0} \in \partial M$. If $q \in \partial M$ is sufficiently close to $q_{0}$, in Fermi coordinates we have $q=q(\eta)=\exp _{q_{0}} \eta$, with $\eta \in \mathbb{R}^{n-1}$. So $v_{q}=v_{\exp _{q_{0}} \eta}$ and we define

$$
\Gamma_{i}=\frac{\partial}{\partial y_{i}} v_{\left.\exp _{q_{0}} \eta\right|_{\eta=0}}
$$

We prove the result for $\Gamma_{1}$, being the other cases completely analogous. By (19) we have that $\Gamma_{1}$ solves

$$
\left\{\begin{array}{cr}
-\Delta \Gamma_{1}=\left[\left.\frac{1}{3} \frac{\partial}{\partial \eta_{1}}\left(\bar{R}_{i j k l}(q(y))\right)\right|_{y=0} y_{k} y_{l}+\left.\frac{\partial}{\partial \eta_{1}}\left(\bar{R}_{n i n j}(q(y))\right)\right|_{y=0}\right] \partial_{i j}^{2} U & \text { on } \mathbb{R}_{+}^{n} \\
\frac{\partial \Gamma_{1}}{\partial t}+n U^{\frac{2}{n-2}} \Gamma_{1}=0 & \text { on } \partial \mathbb{R}_{+}^{n} .
\end{array}\right.
$$

and, since $\frac{\partial R_{n n}}{\partial \eta_{i}}(q)=0$ (see [19, Prop $\left.3.2(4)\right]$ ), we can proceed as at the beginning of this proof to show that $\Gamma_{1}$ exists. Analogously we get the claim for the second derivative.

That concludes the proof. 
Proof of Lemma 6. By (24) we estimate, for some $\theta \in(0,1)$

$$
\begin{gathered}
\tilde{J}_{\varepsilon, \tilde{g}_{q}}\left(W_{\delta, q}+\delta^{2} V_{\delta, q}+\Phi\right)-\tilde{J}_{\varepsilon, \tilde{g}_{q}}\left(W_{\delta, q}+\delta^{2} V_{\delta, q}\right)=\tilde{J}_{\varepsilon, \tilde{g}_{q}}^{\prime}\left(W_{\delta, q}+\delta^{2} V_{\delta, q}\right)[\Phi] \\
+\frac{1}{2} \tilde{J}_{\varepsilon, \tilde{g}_{q}}^{\prime \prime}\left(W_{\delta, q}+\delta^{2} V_{\delta, q}+\theta \Phi\right)[\Phi, \Phi] \\
=\int_{M}\left(\nabla_{\tilde{g}_{q}} W_{\delta, q}+\delta^{2} \nabla_{\tilde{g}_{q}} V_{\delta, q}\right) \nabla_{\tilde{g}_{q}} \Phi+\frac{n-2}{2(n-1)} R_{\tilde{g}_{q}}\left(W_{\delta, q}+\delta^{2} V_{\delta, q}\right) \Phi d \mu_{\tilde{g}_{q}} \\
+\int_{\partial M} \varepsilon \gamma\left(W_{\delta, q}+\delta^{2} V_{\delta, q}\right) \Phi d \sigma_{\tilde{g}_{q}}-(n-2) \int_{\partial M}\left(\left(W_{\delta, q}+\delta V_{\delta, q}\right)^{+}\right)^{\frac{n}{n-2}} \Phi d \sigma_{\tilde{g}_{q}} \\
\quad+\frac{n-2}{2} \int_{\partial M} h_{\tilde{g}_{q}}\left(W_{\delta, q}+\delta^{2} V_{\delta, q}\right) \Phi d \sigma_{\tilde{g}_{q}} \\
+\frac{1}{2} \int_{M}\left|\nabla_{\tilde{g}_{q}} \Phi\right|^{2}+\frac{n-2}{4(n-1)} R_{\tilde{g}_{q}} \Phi^{2} d \mu_{\tilde{g}_{q}}+\frac{1}{2} \int_{\partial M} \varepsilon \gamma \Phi^{2} d \sigma_{\tilde{g}_{q}} \\
+\frac{n-2}{4} \int_{\partial M} h_{\tilde{g}_{q}} \Phi^{2} d \sigma_{\tilde{g}_{q}}-\frac{n}{2} \int_{\partial M}\left(\left(W_{\delta, q}+\delta V_{\delta, q}+\theta \Phi\right)^{+}\right)^{\frac{2}{n-2}} \Phi^{2} d \sigma_{\tilde{g}_{q}} .
\end{gathered}
$$

Immediately we have, by Holder inequality,

$$
\begin{aligned}
& \int_{M}\left|\nabla_{\tilde{g}_{q}} \Phi\right|^{2}+\frac{n-2}{4(n-1)} R_{\tilde{g}_{q}} \Phi^{2} d \mu_{\tilde{g}_{q}}+\int_{\partial M}\left(\varepsilon \gamma+\frac{n-2}{4} h_{\tilde{g}_{q}}\right) \Phi^{2} d \sigma \leq C\|\Phi\|_{\tilde{g}_{q}}^{2}=C\|\tilde{\Phi}\|_{g}^{2} ; \\
& \int_{M} \frac{n-2}{2(n-1)} R_{\tilde{g}_{q}} W_{\delta, q} \Phi d \mu_{\tilde{g}_{q}} \leq C\left\|W_{\delta, q}\right\|_{L^{\frac{2 n}{n+2}}\left(M, \tilde{g}_{q}\right)}\|\Phi\|_{L^{\frac{2 n}{n-2}}\left(M, \tilde{g}_{q}\right)} \leq C \delta^{2}\|\tilde{\Phi}\|_{g} \\
& \delta^{2} \int_{M} a V_{\delta, q} \Phi d \mu_{\tilde{g}_{q}} \leq C \delta^{2}\left\|V_{\delta, q}\right\|_{L^{2}\left(M, \tilde{g}_{q}\right)}\|\Phi\|_{L^{2}\left(M, \tilde{g}_{q}\right)} \leq C \delta^{2}\|\tilde{\Phi}\|_{g} ; \\
& \int_{\partial M} \varepsilon \tilde{\gamma}\left(W_{\delta, q}+\delta^{2} V_{\delta, q}\right) \Phi d \sigma_{\tilde{g}_{q}} \leq C \varepsilon\left\|W_{\delta, q}+\delta V_{\delta, q}\right\|_{L}^{\frac{2(n-1)}{n}\left(\partial M, \tilde{g}_{q}\right)}\|\Phi\|_{L}{ }_{\frac{2(n-1)}{n-2}}\left(\partial M, \tilde{g}_{q}\right) \\
& \leq C \varepsilon \delta\|\tilde{\Phi}\|_{g} \\
& \int_{\partial M}\left(\left(W_{\delta, q}+\delta V_{\delta, q}+\theta \Phi\right)^{+}\right)^{\frac{2}{n-2}} \Phi^{2} d \sigma_{\tilde{g}_{q}} \leq C\left(\left\|W_{\delta, q}+\delta V_{\delta, q}+\theta \Phi\right\|_{L^{\frac{2(n-1)}{n-2}}\left(\partial M, \tilde{g}_{q}\right)}^{\frac{2}{n-2}}\right)\|\Phi\|_{\tilde{g}_{q}}^{2} \\
& \leq C\|\tilde{\Phi}\|_{g}^{2}
\end{aligned}
$$

By integration by parts we have

$$
\begin{aligned}
\int_{M}\left(\nabla_{\tilde{g}_{q}} W_{\delta, q}+\delta^{2} \nabla_{\tilde{g}_{q}} V_{\delta, q}\right) \nabla_{\tilde{g}_{q}} \Phi d \mu_{\tilde{g}_{q}}= & -\int_{M} \Delta_{\tilde{g}_{q}}\left(W_{\delta, q}+\delta^{2} V_{\delta, q}\right) \Phi d \mu_{\tilde{g}_{q}} \\
& +\int_{\partial M}\left(\frac{\partial}{\partial \nu} W_{\delta, q}+\delta^{2} \frac{\partial}{\partial \nu} V_{\delta, q}\right) \Phi d \sigma_{\tilde{g}_{q}} .
\end{aligned}
$$

and, as in (31) we get

$\int_{M} \Delta_{\tilde{g}_{q}}\left(W_{\delta, q}+\delta^{2} V_{\delta, q}\right) \Phi d \mu_{\tilde{g}_{q}} \leq\left\|\Delta_{\tilde{g}_{q}}\left(W_{\delta, q}+\delta^{2} V_{\delta, q}\right)\right\|_{L^{\frac{2 n}{n+2}}\left(M, \tilde{g}_{q}\right)}\|\Phi\|_{\tilde{g}_{q}}=O\left(\delta^{2}\right)\|\tilde{\Phi}\|_{g}$.

Moreover, by Holder inequality,

$$
\int_{\partial M} \delta^{2} \frac{\partial}{\partial \nu} V_{\delta, q} \Phi d \mu_{\tilde{g}_{q}} \leq \delta^{2}\left\|\frac{\partial}{\partial \nu} V_{\delta, q}\right\|_{L^{\frac{2(n-1)}{n}}\left(\partial M, \tilde{g}_{q}\right)}\|\Phi\|_{L^{\frac{2(n-1)}{n-2}}\left(\partial M, \tilde{g}_{q}\right)}=O\left(\delta^{2}\right)\|\tilde{\Phi}\|_{g} .
$$

Since $\partial M$ is umbilic, proceeding as in (33) (34), we get

$$
\int_{\partial M} h_{\tilde{g}_{q}}\left(W_{\delta, q}+\delta^{2} V_{\delta, q}\right) \Phi d \sigma_{\tilde{g}_{q}}=O\left(\delta^{4}\right)\|\tilde{\Phi}\|_{g}
$$


In the end we need to verify that

$$
\begin{aligned}
& \int_{\partial M}\left[(n-2)\left(\left(W_{\delta, q}+\delta^{2} V_{\delta, q}\right)^{+}\right)^{\frac{n}{n-2}}-\frac{\partial}{\partial \nu} W_{\delta, q}\right] \Phi d \sigma_{\tilde{g}_{q}} \\
& =\left\|(n-2)\left(\left(W_{\delta, q}+\delta^{2} V_{\delta, q}\right)^{+}\right)^{\frac{n}{n-2}}-\frac{\partial}{\partial \nu} W_{\delta, q}\right\|_{L}{ }_{L}^{\frac{2(n-1)}{n}}\left(\partial M, \tilde{g}_{q}\right) \\
& \|\Phi\|_{L}^{\frac{2(n-1)}{n-2}}\left(\partial M, \tilde{g}_{q}\right) \\
& =o\left(\delta^{2}\right)\|\tilde{\Phi}\|_{g}
\end{aligned}
$$

In fact, by (16) and by taylor expansion we have

$$
\begin{gathered}
\int_{\partial M}\left[(n-2)\left(\left(W_{\delta, q}+\delta^{2} V_{\delta, q}\right)^{+}\right)^{\frac{n}{n-2}}-\frac{\partial}{\partial \nu} W_{\delta, q}\right]^{\frac{2(n-1)}{n}} d \sigma_{\tilde{g}_{q}} \\
\leq \int_{\partial \mathbb{R}_{+}^{n}}\left[(n-2)\left(\left(U_{\delta}+\delta^{2}\left(v_{q}\right)_{\delta}\right)^{+}\right)^{\frac{n}{n-2}}+\frac{\partial}{\partial t} U_{\delta}\right]^{\frac{2(n-1)}{n}} d z+o\left(\delta^{\frac{4(n-1)}{n}}\right) \\
\leq \int_{\partial \mathbb{R}_{+}^{n}}\left[n\left(\left(U_{\delta}+\theta \delta^{2}\left(v_{q}\right)_{\delta}\right)^{+}\right)^{\frac{2}{n-2}} \delta^{2}\left(v_{q}\right)_{\delta}\right]^{\frac{2(n-1)}{n}} d z+o\left(\delta^{\frac{4(n-1)}{n}}\right)=o\left(\delta^{\frac{4(n-1)}{n}}\right),
\end{gathered}
$$

which concludes the proof.

\section{REFERENCES}

[1] S. Almaraz, A compactness theorem for scalar-flat metrics on manifolds with boundary, Calc. Var. 41 (2011) 341-386

[2] S. Almaraz, Blow-up phenomena for scalar-flat metrics on manifolds with boundary, J. Differential Equations 251 (2011), no. 7, 1813-1840.

[3] S. Almaraz, An existence theorem of conformal scalar-flat metrics on manifolds with boundary. Pacific J. Math. 248 (2010), 1-22.

[4] T. Aubin, Equations differentielles non lineaires et probleme de Yamabe concernant la courbure scalaire, J. Math. Pures Appl. 55 (1976), 269-296

[5] T. Aubin, Some Nonlinear Problems in Riemannian Geometry. Springer Monographs in Mathematics. Springer, Berlin (1998).

[6] S. Brendle and F. C. Marques, Recent progress on the Yamabe problem, in: Surveys in geometric analysis and relativity, Adv. Lect. Math. (ALM) 20, International Press, Somerville (2011), 29-47.

[7] M.M. Disconzi, M.A. Khuri, Compactness and non-compactness for the Yamabe problem on manifolds with boundary, J. Reine Angew. Math. 724 (2017) 145-201.

[8] O. Druet, Compactness for Yamabe metrics in low dimensions, Int. Math. Res. Not. 23 (2004), 1143-1191.

[9] O. Druet, E. Hebey, Blow-up examples for second order elliptic PDEs of critical Sobolev growth, Trans. Amer. Math. Soc. 357 (2005), no. 5, 1915-1929

[10] J. F. Escobar, Conformal deformation of a Riemannian metric to a scalar flat metric with constant mean curvature on the boundary. Ann. of Math. 136 (1992), 1-50.

[11] J. F. Escobar, The Yamabe problem on manifolds with boundary, J. Differential Geometry 35 (1992), no. 1, 21-84.

[12] P. Esposito, A. Pistoia Blowing-up solutions for the Yamabe equation, Port. Math. 71 (2014), 249-276.

[13] P. Esposito, A. Pistoia, J. Vetois, The effect of linear perturbations on the Yamabe problem, Math. Ann. 358 (2014), no. 1-2, 511-560

[14] V. Felli, M. Ould Ahmedou, Compactness results in conformal deformations of Riemannian metrics on manifolds with boundaries, Math. Z. 244 (2003), 175-210.

[15] M.G. Ghimenti, A.M. Micheletti, A. Pistoia, On Yamabe type problems on Riemannian manifolds with boundary, Pacific Journal of Math. 284 (2016), 79-102.

[16] M.G. Ghimenti, A.M. Micheletti, A. Pistoia, Linear Perturbation of the Yamabe Problem on Manifolds with Boundary, J. Geom. Anal., in press, arxiv https://arxiv.org/abs/1611.01336.

[17] S. Kim, M. Musso, J. Wei, Existence theorems of the fractional Yamabe problem. Anal. PDE 11 (2018), 75-113.

[18] M. Khuri, F. Marques, R. Schoen, A compactness theorem for the Yamabe problem, J. Differential Geom. 81 (2009), 143-196. 
[19] F. Marques, Existence results for the Yamabe problem on manifolds with boundary, Indiana Univ. Math. J. 54 (2005), 1599-1620.

[20] F. C. Marques, Conformal deformations to scalar-flat metrics with constant mean curvature on the boundary, Comm. Analysis Geom. 15 (2007), 381-405.

[21] A.M. Micheletti, A. Pistoia, The role of the scalar curvature in a nonlinear elliptic problem on Riemannian manifolds, Calc. Var. 34 (2009), 233-265.

[22] A.M. Micheletti, A. Pistoia, J. Vetois, Blow-up Solutions for Asymptotically Critical Elliptic Equations on Riemannian Manifolds, Indiana Univ. Math. Journal 58 (2009), 1719-1746.

[23] P. Morabito, A. Pistoia, G. Vaira, Towering phenomena for the Yamabe equation on symmetric manifolds, Potential Anal. 47, 53-102 (2017)

[24] A. Pistoia, G. Vaira, Clustering phenomena for linear perturbation of the Yamabe equation, to appear on J. Lond. Math. Soc. 1-13.

[25] F. Robert, J. Vetois, Examples of non-isolated blow-up for perturbations of the scalar curvature equation on non-locally conformally flat manifolds, J. Differential Geom. 98, 349-356 (2014)

[26] R. Schoen, Conformal deformation of a Riemannian metric to constant scalar curvature, J. Differential Geometry 20 (1984), 479-495

[27] N. Trudinger, Remarks concerning the conformal deformation of Riemannian structures on compact manifolds, Annali Scuola Norm. Sup. Pisa 22 (1968), 265-274.

[28] H. Yamabe, On a deformation of Riemannian structures on compact manifolds, Osaka Math. J. 12 (1960), 21-37.

(Marco Ghimenti) Dipartimento di Matematica Università di Pisa Largo Bruno PonTECORVo 5, I - 56127 Pisa, Italy

E-mail address: marco.ghimenti@unipi.it

(Anna Maria Micheletti) Dipartimento di Matematica Università di Pisa Largo Bruno Pontecorvo 5, I - 56127 Pisa, Italy

E-mail address: a.micheletti@dma.unipi.it

(Angela Pistoia) Dipartimento SBAi, Universtà di Roma "La Sapienza", via Antonio Scarpa 16, 00161 Roma, Italy

E-mail address: angela.pistoia@uniroma1.it 